\title{
In utero exposure to $\Delta 9$-tetrahydrocannabinol leads to postnatal catch-up growth and dysmetabolism in the adult rat liver
}

\author{
Shelby L. Oke ${ }^{1,2}$, Kendrick Lee ${ }^{1,2}$, Rosemary Papp ${ }^{1}$, Steven R. Laviolette ${ }^{3}$ and Daniel B. Hardy ${ }^{1-4 *}$ \\ a1 Department of Physiology and Pharmacology, Schulich School of Medicine and Dentistry, Western Univer- \\ sity, 1151 Richmond Street, London, Ontario, Canada, N6A 5C1 \\ 2 The Children's Health Research Institute, The Lawson Health Research Institute, London, Ontario, Canada, \\ N6A 5C1 \\ 3 Department of Anatomy and Cell Biology, Schulich School of Medicine and Dentistry, Western University, \\ 1151 Richmond Street, London, Ontario, Canada, N6A 5C1 \\ 4 Department of Obstetrics and Gynaecology, Schulich School of Medicine and Dentistry, Western University, \\ 1151 Richmond Street, London, Ontario, Canada, N6A 5C1 \\ * Correspondence: daniel.hardy@schulich.uwo.ca
}

\begin{abstract}
Rates of gestational cannabis use have increased despite limited evidence for its safety in fetal life. Recent animal studies demonstrate that prenatal exposure to $\Delta 9$-tetrahydrocannabinol $(\Delta 9$ THC, the psychoactive component of cannabis) promotes intrauterine growth restriction (IUGR), culminating in postnatal metabolic deficits. Given IUGR is associated with impaired hepatic function, we hypothesized that $\triangle 9$-THC offspring would exhibit hepatic dyslipidemia. Pregnant Wistar rat dams received daily injections of vehicular control or $3 \mathrm{mg} / \mathrm{kg} \Delta 9-\mathrm{THC}$ i.p. from embryonic day (E) 6.5 through E22. Exposure to $\Delta 9$-THC decreased the liver to body weight ratio at birth, followed by catch-up growth by three weeks of age. At six months, $\Delta 9$-THC-exposed male offspring exhibited increased visceral adiposity and higher hepatic triglycerides. This was instigated by augmented expression of enzymes involved in triglyceride synthesis (ACC $\alpha$, SCD, FABP1, and DGAT2) at three weeks. Furthermore, the expression of hepatic DGAT1/DGAT2 was sustained at six months, concomitant with mitochondrial dysfunction (i.e., elevated p66shc) and oxidative stress. Interestingly, decreases in miR-203a-3p and miR-29a/b/c, both implicated in dyslipidemia, was also observed in these $\Delta 9$-THC-exposed offspring. Collectively, these findings indicate that prenatal $\Delta 9-\mathrm{THC}$ exposure results in long-term dyslipidemia associated with enhanced hepatic lipogenesis. This is attributed by mitochondrial dysfunction and epigenetic mechanisms.
\end{abstract}

Keywords: $\Delta 9$-tetrahydrocannabinol, intrauterine growth restriction, liver, metabolism, triglycerides, oxidative stress, mitochondria, miR-203a-3p, miR-29a/b/c

\section{Introduction}

Cannabis is the most commonly used recreational drug among individuals of reproductive age. In 2019, the Canadian Cannabis Survey found that approximately $21 \%$ of female adolescents and young adults self-reported daily or almost daily use of cannabis, while $40 \%$ reported monthly use [1]. The American College of Obstetricians and Gynecologists has further found that 2-5\% of individuals use cannabis during pregnancy, and this was increased to 15-28\% among young women who lived in urban settings and whom were socioeconomically disadvantaged [2]. With the recent legalization of recreational cannabis in Canada and select American states, the use of cannabis during pregnancy has become increasingly popular despite limited evidence of its safety. Many pregnant individuals use cannabis to diminish nausea, anxiety and depression, as they believe it to be a 'safe' and natural alternative to prescription medications [3-5]. Alarmingly, gestational exposure to cannabis can increase the risk for adverse neonatal outcomes, including low birth weight and preterm delivery [6-8]. However, these studies are confounded by that fact that cannabis users are more likely to concurrently abuse other drugs such as alcohol and tobacco [9], highlighting the need 
for animal studies that focus on the effects of specific constituents of cannabis (i.e., $\Delta 9$ tetrahydrocannabinol $[\triangle 9-\mathrm{THC}]$ and cannabidiol $[\mathrm{CBD}])$ on short- and long-term maternal-fetal outcomes [9].

Cannabis is composed of several distinct compounds that stimulate the endocannabinoid system via interaction with cannabinoid receptor type 1 (CB1R) and CB2R. These receptors are highly expressed throughout the central nervous system (CNS); therefore, their activation by cannabinoids has profound effect on mood, pain, memory and appetite [10]. At the same time, CB1R and CB2R are localized in peripheral metabolic tissues, suggesting that the endocannabinoid system has additional roles outside of neurocognitive function [11-14]. Many cannabinoids are also lipophilic and able to cross the placental barrier, thereby entering fetal circulation and allowing for activation of CB1R and CB2R [15]. While activation of the endocannabinoid system is essential for the maintenance of pregnancy, the presence of exogenous cannabinoids can also directly interfere with fetal growth and development through the disruption of endocannabinoid signalling. Gestational exposure to $\triangle 9$-THC, the principal psychoactive component of cannabis, has been demonstrated to promote placental insufficiency and symmetrical intrauterine growth restriction (IUGR) in rodents, characterized by low birth weight and reduced neonatal organ weight [16-21]. Of note, the liver to body weight ratio is reduced in $\triangle 9$-THC offspring, followed by rapid catch-up growth in the first three weeks of life [21]. While the developmental origins of health and disease (DOHaD) postulates that there is an inverse relationship between birth weight and metabolic health, the role of gestational $\Delta 9$-THC exposure on postnatal hepatic function has not yet been investigated.

The liver has a critical role in controlling lipid metabolism, which involves the synthesis and degradation of structural and functional lipid molecules. Naturally, impaired liver function leads to dyslipidemia, whereby triglycerides and cholesterol become elevated in the liver and plasma. Dyslipidemia is a well characterized attribute of the metabolic syndrome that often accompanies obesity; therefore, individuals with either of these conditions exhibit an increased risk for developing diabetes and cardiovascular disease [22]. Moreover, epidemiological studies have identified that low birth weight individuals with decreased liver to body weight ratio are more likely to be obese and have non-alcoholic fatty liver disease (NAFLD) during childhood and adult life [23-29]. Similar trends have been found in studies of rodent IUGR offspring; we have previously shown that maternal nicotine exposure leads to elevated hepatic and circulating triglycerides in adult male offspring, while protein-restricted adult males have increased hepatic and circulating cholesterol [30,31]. Hepatic hyperlipidemia often occurs due to divergent synthesis and metabolism of free fatty acids, which can be of dietary, circulating or de novo origin [32]. In particular, de novo lipogenesis is a highly regulated, multi-step process that involves the esterification of fatty acids to triglycerides through the action of numerous enzymes. Initially, acetyl-coA carboxylase (ACC $\alpha$ ) catalyzes the carboxylation of acetyl-coA to malonyl-coA, which acts as a substrate for fatty acid synthase (FAS) in generating saturated fatty acids [32,33]. Stearoyl-coA desaturase-1 (SCD-1) then converts these saturated fatty acids into monounsaturated fatty acids (MUFAs), which undergo various elongation reactions to generate the long chain fatty acids that feed into triglyceride synthesis pathways [34]. The cytosolic transport of these long chain fatty acids is mediated by various fatty acid binding proteins (FABPs), which are highly expressed in tissues that control lipid metabolism. Importantly, the diacylglycerol acyltransferase (DGAT) enzyme catalyzes the terminal step of triglyceride synthesis $[35,36]$. Studies of the growth-restricted liver demonstrate that many of these enzymes are particularly sensitive to developmental reprogramming, as their abundance and activity levels change with exposure to a variety of gestational insults leading to long-term dyslipidemia [30,3739]. That said, the underlying mechanisms linking a poor in utero environment to these metabolic deficits remain elusive.

Oxidative stress and mitochondrial dysfunction occur with many metabolic pathologies, including hepatic dyslipidemia. Many studies have shown that growth-restricted offspring exhibit oxidative stress and impaired mitochondrial metabolism in the liver [40-45]; therefore, it is possible that mitochondrial dysfunction precedes dyslipidemia in IUGR offspring. This may be mediated, in part, by the adaptor protein p66Shc, which is known to accelerate the mitochondrial production of 
ROS [46]. Human and animal studies indicate that the p66Shc-induced oxidative signal is involved in the accumulation of intracellular lipids, leading to obesity and NAFLD [47,48]. Not surprisingly, growth-restricted offspring display increased levels of p66Shc in the liver, kidney, and pancreas at birth and in postnatal life $[45,49,50]$. Furthermore, using a rodent model of maternal protein restriction, we have also shown that adult male offspring have increased hepatic protein abundance of p66Shc following postnatal catch-up growth [45]. These same offspring also have aberrant expression of various microRNAs (miRs) in the liver, so it's possible that the expression of genes involved in de novo lipogenesis and mitochondrial function may be regulated via epigenetic mechanisms [51]. Knowing that gestational exposure to $\Delta 9$-THC leads to compromised liver growth, the present study investigates the effects of $\Delta 9$-THC on hepatic lipid metabolism in the exposed offspring. Given the role of mitochondria in metabolic disease and the growth-restricted liver, we hypothesized that $\triangle 9$-THC-exposed offspring would exhibit oxidative stress and mitochondrial dysfunction, along with aberrant expression of miRs that are known to result in dysmetabolism.

\section{Results}

\subsection{Gestational exposure to $\triangle 9-T H C$ leads to hepatic catch-up growth by three weeks of age}

At birth (i.e., PND 1), $\triangle 9$-THC-exposed offspring exhibited decreased liver to body weight ratio compared to control offspring (Table $1 ; p<0.05$ ). It should be noted that in this same cohort of vehicle and $\triangle 9$-THC offspring, we have published that $\triangle 9$-THC exposure during pregnancy did not lead to changes in maternal food intake, maternal weight gain, litter size or gestational length [21]. By three weeks of age, these offspring had exhibited hepatic catch-up growth, as there were no significant differences between liver to body weight ratios of $\Delta 9$-THC-exposed offspring and control offspring (Table 1). This is consistent with other rodent models of IUGR, including maternal nicotine exposure and maternal protein restriction, whereby growth-restricted offspring also undergo catchup growth by three weeks [31,52]. At three weeks of age, there were also no differences in liver to body weight ratios between male and female offspring of either treatment group (Table 1), indicating that there were no sex-specific effects of gestational $\triangle 9$-THC with respect to hepatic catch-up growth. At six months of age, liver to body weight ratio remained equal between both groups and sexes (Table 1). It should also be noted that gestational exposure to $\triangle 9$-THC did not significantly affect postnatal food intake (PND50-60) in either males (vehicle, $19.3 \pm 1.5 \mathrm{~g}$ food/day/offspring; $\triangle 9-\mathrm{THC}$, $15.12 \pm 2.1 \mathrm{~g}$ food/day/offspring) or females (vehicle, $17.7 \pm 1.6 \mathrm{~g}$ food/day/offspring; $\Delta 9-\mathrm{THC}, 20.03$ $\pm 1.9 \mathrm{~g}$ food/day/offspring.

\subsection{Adult 49 -THC-exposed offspring exhibit elevated visceral adiposity and hepatic dyslipidemia} following gestational exposure to $\triangle 9-T H C$

As mentioned previously, adult IUGR offspring are vulnerable to the development of obesity and hepatic pathologies involved in lipid storage and metabolism. Given that $\Delta 9-\mathrm{THC}$ induces symmetrical IUGR [21], we were interested in comparing the levels of visceral adipose tissue and hepatic lipids between control and $\triangle 9$-THC-exposed offspring during adult life. At six months, offspring exposed to gestational $\triangle 9$-THC exhibited increased visceral adipose to body weight ratio (Table $1 ; p<0.05)$, suggesting dyslipidemia in these animals. That said, this was true only when examining both sexes together, as there were differences in visceral adipose to body weight ratio 
Table 1. Gestational exposure to $\Delta 9$-tetrahydrocannabinol ( $\triangle 9$-THC) leads to hepatic catch-up growth by three weeks and increased visceral adiposity in adult life.

\begin{tabular}{|c|c|c|c|c|c|}
\hline & $\begin{array}{c}\text { Treatment } \\
\text { Group }\end{array}$ & Sex & Birth & Three Weeks & Six Months \\
\hline \multirow{6}{*}{$\begin{array}{l}\text { Liver to Body } \\
\text { Weight Ratio }\end{array}$} & \multirow{3}{*}{ Control } & Male & & $0.0458^{a} \pm 0.0026$ & $0.0319^{a} \pm 0.0024$ \\
\hline & & Female & & $0.0445^{a} \pm 0.0017$ & $0.0354^{a} \pm 0.0034$ \\
\hline & & Both sexes & $0.0392^{\#} \pm 0.0039$ & $0.0451^{\sharp} \pm 0.0011$ & $0.0333^{\sharp} \pm 0.0020$ \\
\hline & \multirow{3}{*}{$\triangle 9-\mathrm{THC}$} & Male & & $0.0459^{a} \pm 0.0010$ & $0.0350^{\mathrm{a}} \pm 0.0017$ \\
\hline & & Female & & $0.0437^{a} \pm 0.0009$ & $0.0351^{\mathrm{a}} \pm 0.0022$ \\
\hline & & Both sexes & $0.0293^{•} \pm 0.0018$ & $0.0445^{\sharp} \pm 0.0020$ & $0.0351^{\#} \pm 0.0014$ \\
\hline \multirow{6}{*}{$\begin{array}{c}\text { Adipose to Body } \\
\text { Weight Ratio }\end{array}$} & \multirow{3}{*}{ Control } & Male & & & $0.01685^{\mathrm{a}} \pm 0.0015$ \\
\hline & & Female & & & $0.0202^{\mathrm{a}} \pm 0.0025$ \\
\hline & & Both sexes & & & $0.01828^{\#} \pm 0.0014$ \\
\hline & \multirow{3}{*}{$\Delta 9-\mathrm{THC}$} & Male & & & $0.0207^{a} \pm 0.0020$ \\
\hline & & Female & & & $0.0234^{a} \pm 0.0022$ \\
\hline & & Both sexes & & & $0.0223^{\star} \pm 0.0015$ \\
\hline
\end{tabular}

Liver growth and visceral adipose deposition were assessed for all offspring by calculating liver to body weight ratio and adipose to body weight ratio. All data are expressed as means \pm SEM ( $n=7-18 /$ sex/group). The effects of $\Delta 9$-THC on liver to body weight ratio and visceral adipose to body weight ratio were determined via Student's two-tailed unpaired t-test. Sex-specific differences in liver to body weight ratio were assessed using a two-way ANOVA followed by a Holm-Sidak-corrected multiple comparisons test. Groups labelled with different letters or symbols are significantly different from each other.

when examining each sex individually. To explore this further, we measured hepatic and circulating triglyceride and cholesterol levels of each sex for both groups at six months of age. While both hepatic and circulating triglyceride and cholesterol levels of $\Delta 9$-THC-exposed female offspring remained unchanged at six months (Figures $1 \mathrm{~A}-\mathrm{D}$ ), hepatic triglycerides were elevated in $\triangle 9$-THC-exposed males in comparison to control males and all females (Figure $1 \mathrm{~A} ; p<0.05$ ). Interestingly, hepatic cholesterol was unaltered in six-month old male offspring exposed to gestational $\triangle 9$-THC, as were circulating triglycerides and cholesterol (Figures 1B-D). Since the differences in lipid content were observed exclusively in livers of male offspring, we further quantified hepatic triglycerides and cholesterol in male offspring at an earlier timepoint. At three weeks of age, hepatic triglycerides and cholesterol were not significantly altered between control and $\Delta 9$-THC-exposed male offspring (Figures 1E and 1F).

2.3. Elevated hepatic triglyceride levels coincide with increased diglycerol acyltransferase and p66Shc protein levels in the livers of adult male 49 -THC-exposed offspring

To gain insight on possible mechanisms involved in the elevation of hepatic triglycerides among adult male $\Delta 9$-THC-exposed offspring, we performed western immunoblotting for enzymes involved in the triglyceride synthesis pathway. $\triangle 9$-THC-exposed males exhibited increased DGAT1 (Figure 2F; $p<0.05$ ) and DGAT2 (Figure 2G; $p<0.05$ ) in the liver at six months of age, while there were no significant differences in hepatic ACC $\alpha$, FAS, SCD, or FABP1 (Figures 2B-E). Adult female $\Delta 9$ THC-exposed offspring also demonstrated a significant increase in DGAT2 (Figure $2 \mathrm{~N} ; p<0.05$ ) relative to female controls, while all other enzymes involved in triglyceride synthesis were unchanged (Figures 2I-M). As p66Shc is linked to the accumulation of intracellular lipids [47], we further quantified the relative abundance of p66Shc protein in control and $\triangle 9$-THC-exposed offspring. Adult male offspring exposed to gestational $\triangle 9$-THC demonstrated increased hepatic p66Shc protein abundance at 6 months compared to control male offspring (Figure 2H; $p<0.05$ ), while p66Shc was unchanged in adult female offspring (Figure 2O). Again, because changes were observed 
A

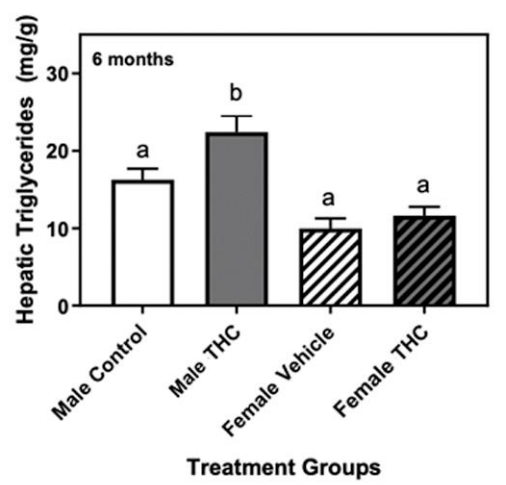

D

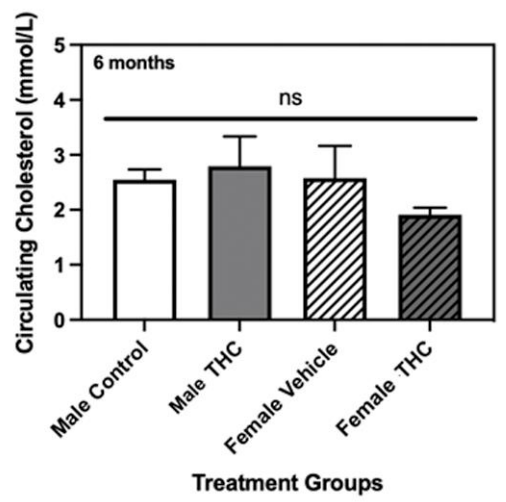

B

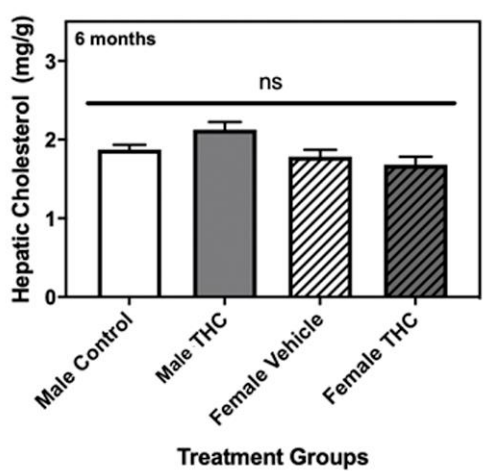

E

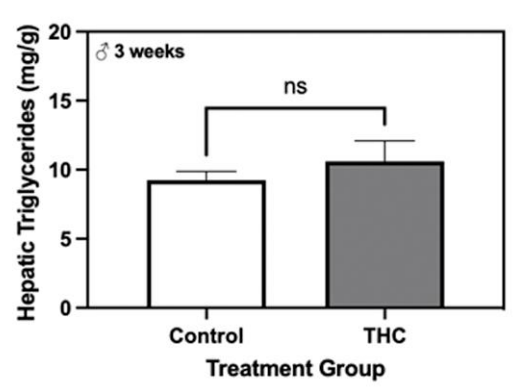

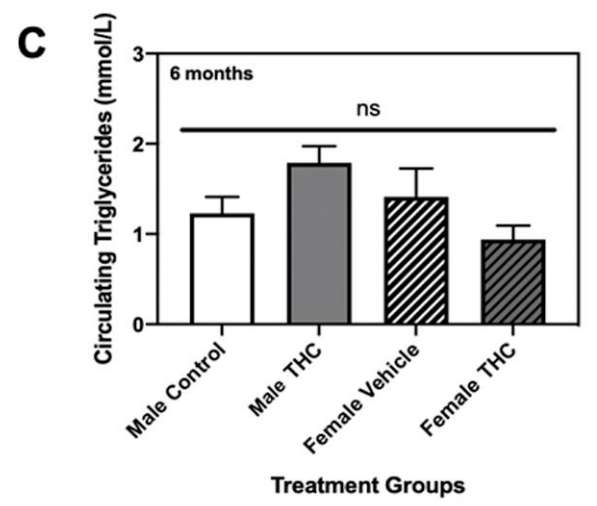

$\mathbf{F}$

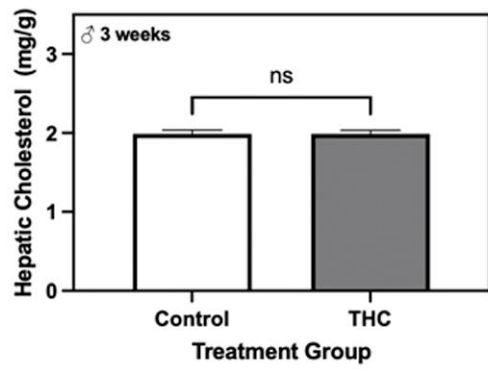

Figure 1. The effects of gestational $\Delta 9$-tetrahydrocannabinol $(\Delta 9-\mathrm{THC})$ on triglyceride and cholesterol levels in the liver and plasma of exposed offspring at three weeks and six months of age. At six months of age, hepatic triglycerides (A) and cholesterol (B) were assessed in both male and female offspring (mg of lipid/g of tissue), along with circulating levels of triglycerides (C) and cholesterol (D; mmol/L). Hepatic triglycerides (E) and cholesterol (F) were also quantified in three-week old male offspring. Data are expressed as the mean \pm SEM. The effects of $\triangle 9$-THC in three-week old male offspring were determined via Student's two-tailed unpaired ttest, while six-month old offspring results were analyzed using a two-way ANOVA followed by a Holm-Sidak-corrected multiple comparisons test. Groups labelled with different letters are significantly different from each other $(p<0.05)$.

in hepatic lipid content exclusively in adult male offspring, we further examined the expression of these enzymes in males at three weeks of age. At three weeks, $\Delta 9$-THC-exposed male offspring had increased protein abundance of DGAT2 (Figure 3G; $p<0.001$ ) and FABP1 (Figure 3E; $p<0.01$ ). However, protein levels of ACC $\alpha$, FAS, SCD, DGAT1 and p66Shc were unchanged in male offspring at three weeks of age (Figures 3B-D, F and H).

\subsection{Gestational exposure to $\triangle 9-T H C$ does not affect protein levels of enzymes involved in aerobic metabolism at six months of age}

Given that male offspring exhibit altered lipid metabolism and increased p66Shc, which are both implicated in mitochondrial dysfunction [53,54], we next assessed protein levels of enzymes involved in aerobic metabolism. At six months, the abundance of mitochondrial transcription factor A (TFAM), a protein that is essential in activating mitochondrial genome transcription, was unaltered in both male and female offspring exposed to gestational $\triangle 9-\mathrm{THC}$ (Figures $4 \mathrm{~B}$ and $4 \mathrm{~F}$ ). Furthermore, male offspring exhibited no changes in the ratio of phosphorylated pyruvate dehydrogenase at serine residue 232 (p-PDH[Ser232]) to total $\mathrm{PDH}$, lactate dehydrogenase subunit A (LDHa), or citrate synthase (Figures 4C-E). Interestingly, six-month female offspring exposed to gestational $\triangle 9$-THC displayed a decrease in the ratio of p-PDH[Ser232] to total PDH (Figure 4G), while protein levels of LDHa and citrate synthase were unchanged (Figures $4 \mathrm{H}$ and $4 \mathrm{I})$. However, at three weeks of age, male $\Delta 9$-THC offspring exhibited increased levels of TFAM (Figure $5 \mathrm{~B} ; p<0.01$ ) 
A

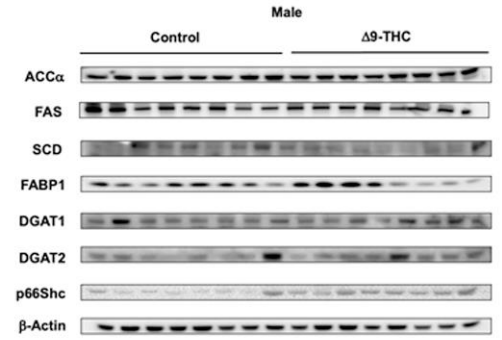

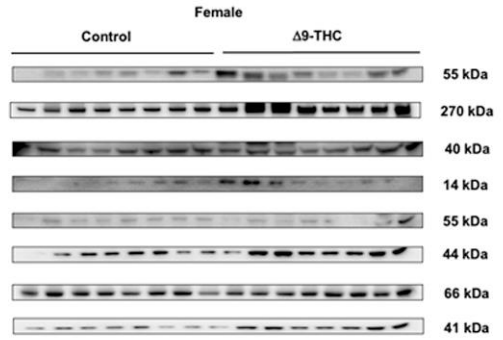

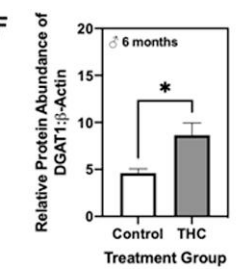

Control THC
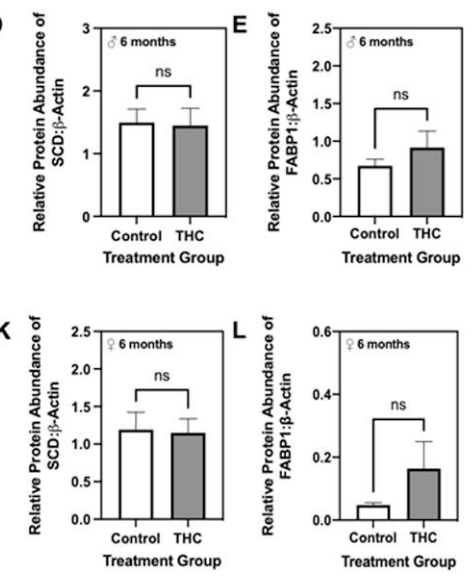

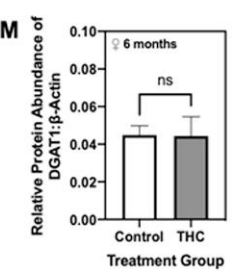

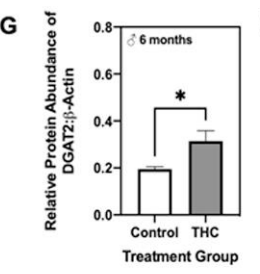
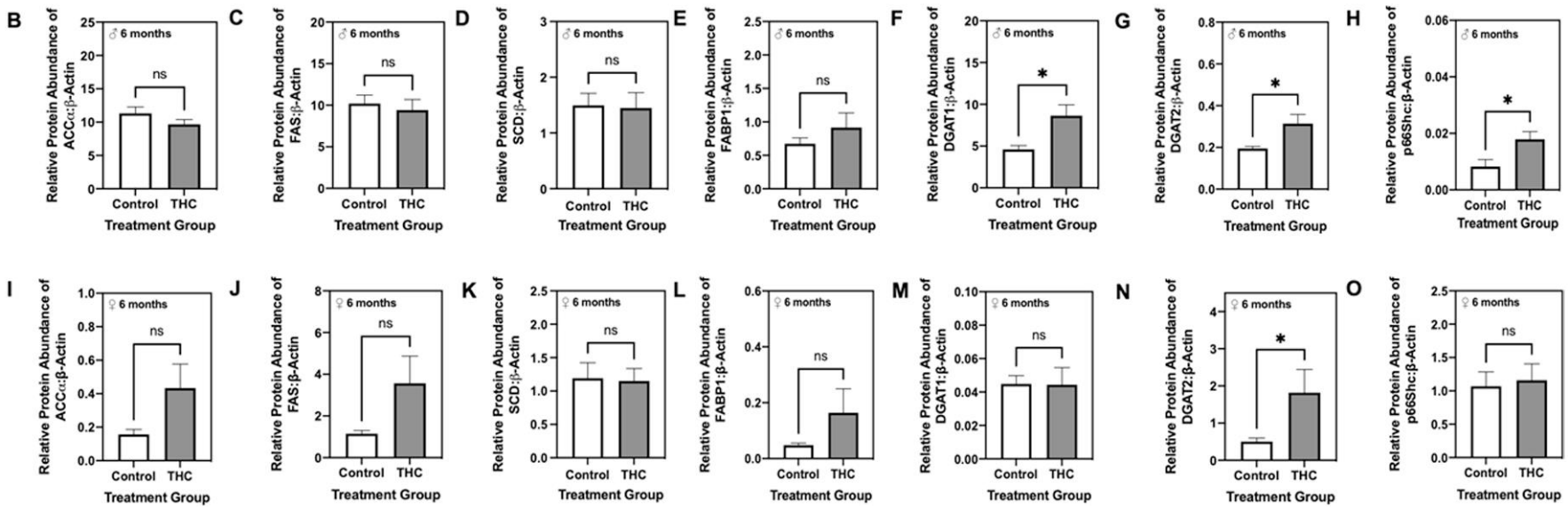

Figure 2. Gestational exposure to $\Delta$ 9-tetrahydrocannabinol ( $\triangle 9$-THC) leads to upregulation of lipogenic enzymes in male offspring at six months of age. (A) Representative western immunoblots illustrating hepatic expression of acetyl-coA carboxylase (ACC $\alpha$ ), fatty acid synthase (FAS), stearoyl-coA desaturase (SCD), fatty acid binding protein 1 (FABP1), diacylglycerol acyltransferase (DGAT) 1, DGAT2, and p66Shc in male and female offspring at six months of age. Protein abundances of each enzyme for male offspring (B$\mathrm{H})$ and female offspring (I-O) were normalized to $\beta$-Actin \pm SEM ( $n=7-8 /$ group). All protein abundances were analyzed using a twotailed unpaired Student's t-test. *Significant difference $(p<0.05)$.

and LDHa (Figure 5D; $p<0.01$ ), while the ratio of $\mathrm{p}-\mathrm{PDH}[$ Ser232] to total $\mathrm{PDH}$ and levels of citrate synthase were unchanged (Figures $5 \mathrm{C}$ and $5 \mathrm{E}$ ).

\subsection{Gestational exposure to $\triangle 9$-THC leads to altered hepatic protein levels of superoxide dismutase}

1 in adult male offspring

Given that increased p66Shc is also associated with oxidative stress[46], we further analyzed the abundance of antioxidant enzymes that are critical in combatting the damaging effects of ROS. Male offspring demonstrated decreased protein levels of SOD1 at six months of age (Figure 6C; $p<0.05$ ), while catalase and SOD2 remained unchanged at this time point (Figures 6B and 6D). Lipid peroxidation was also unaffected at six months of age in male offspring exposed to $\Delta 9-\mathrm{THC}$, as indicated by levels of 4-hydroxynonenol (4HNE; Figure 6E). At three weeks, male $\Delta 9$-THC-exposed offspring did not exhibit any changes in catalase, superoxide dismutase (SOD) 1 or SOD2 protein levels (Figures 6F-H); however, hepatic lipid peroxidation was increased as indicated by elevated levels of $4 \mathrm{HNE}$ (Figure 6I; $p<0.05$ ).

\subsection{Mitochondrial electron transport chain complexes are increased in male offspring at six months and three weeks of age following gestational exposure to $\triangle 9$-THC}

The mitochondrial electron transport chain is known to have significant contribution to the production of ROS, particularly through the activity of complexes I and III [55]. Given we observed changes in antioxidant and mitochondrial proteins in $\triangle 9$-THC offspring, we next quantified protein levels of each subunit of the electron transport chain. At six months, male offspring exposed to gestational $\triangle 9$-THC exhibited increased abundance of complexes I, III, and V (Figures 7B, 7D and 7F; $p<0.05$ ), while levels of complexes II and IV were unchanged (Figures 7C and 7E). While adult female 


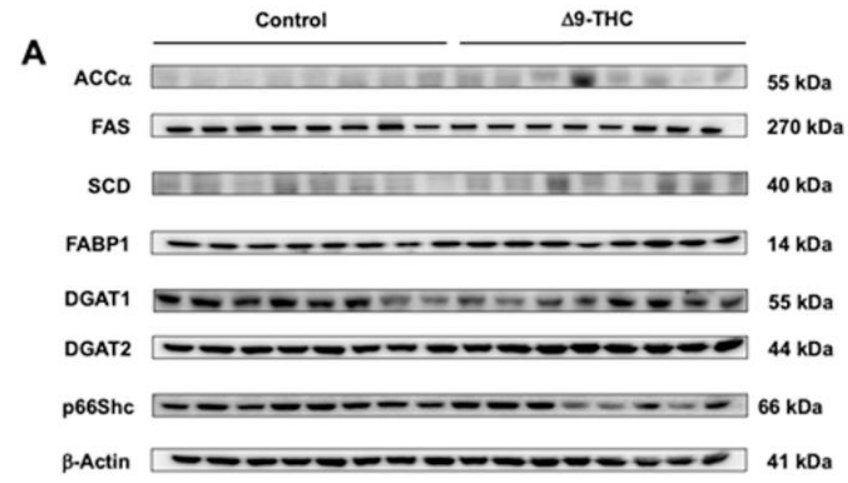

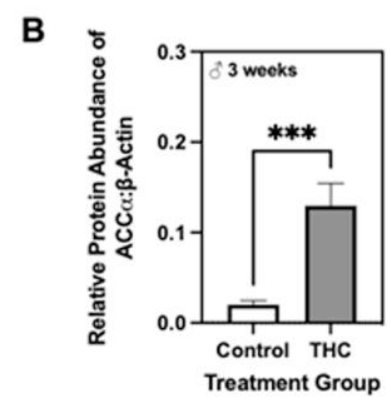

$\mathbf{F}$

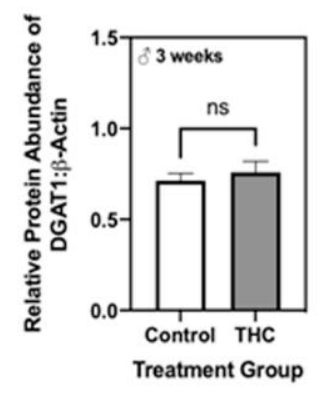

C

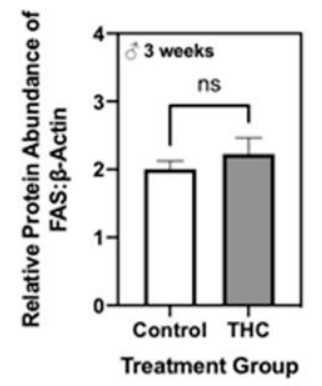

G

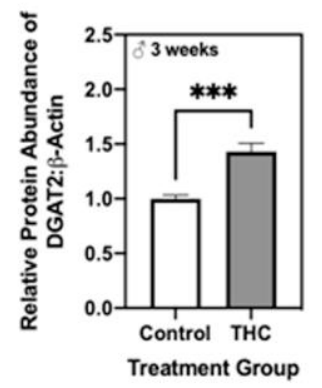

D

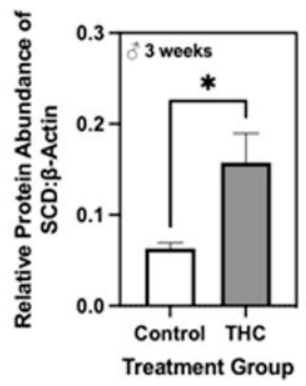

H

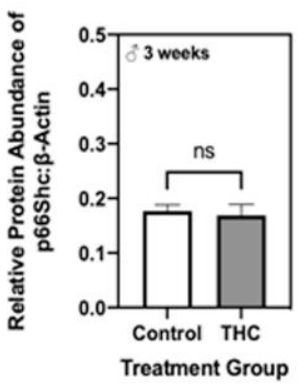

E

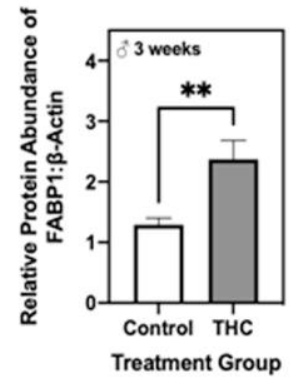

Figure 3. Three-week old male offspring exhibit increased lipogenic enzyme expression in the liver following gestational exposure to $\Delta$ 9-tetrahydrocannabinol ( $\triangle 9$-THC). (A) Representative western immunoblots illustrating hepatic expression of acetyl-coA carboxylase (ACC $\alpha$ ), fatty acid synthase (FAS), stearoyl-coA desaturase (SCD), fatty acid binding protein 1 (FABP1), diacylglycerol acyltransferase (DGAT) 1, DGAT2, and p66Shc in male offspring at three weeks of age. Protein abundances of (B) ACC $\alpha,(C)$ FAS, (D) SCD, (E) FABP1, (F) DGAT1, (G) DGAT2 and (H) p66Shc were normalized to $\beta$-Actin \pm SEM (n= 7-8/group). All protein abundances were analyzed using a two-tailed unpaired Student's t-test. ${ }^{*}$ Significant difference $(p<0.05),{ }^{* *}$ significant difference $(p<0.01),{ }^{* * *}$ significant difference $(p<0.001)$.

offspring demonstrated an increase in the protein abundance of complex I (Figure 7G; $p<0.01$ ), the expression of all other subunits remained unchanged (Figures $7 \mathrm{H}-\mathrm{K}$ ). At three weeks, male $\Delta 9-\mathrm{THC}$ exposed offspring also demonstrated increased levels of complexes I and III (Figures 8B and 8D and $\mathrm{X} ; p<0.05$ ), while all other complexes were unchanged (Figures $8 \mathrm{C}, 8 \mathrm{E}$ and $8 \mathrm{~F}$ ).

\subsection{Adult male offspring exposed to gestational $\triangle 9$-THC exhibit decreased hepatic transcript levels} of $m i R-203 a-3 p$ and $m i R-29 a / b / c$

Various miRs are known to be dysregulated with metabolic disease, and we have previously shown that IUGR offspring with postnatal catch-up growth exhibit altered expression of miRs, specifically, miR-29a/b/c [51]. To better understand the potential mechanisms behind the increase in p66Shc protein levels among adult male $\Delta 9$-THC-exposed livers, we first investigated whether hepatic transcript abundance of miR-203a-3p, which silences p66shc [56], was altered in 

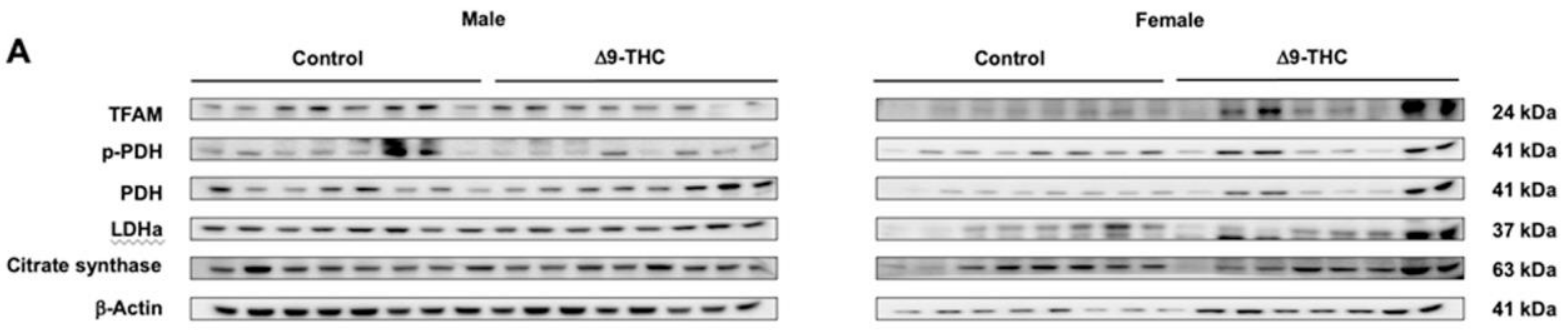

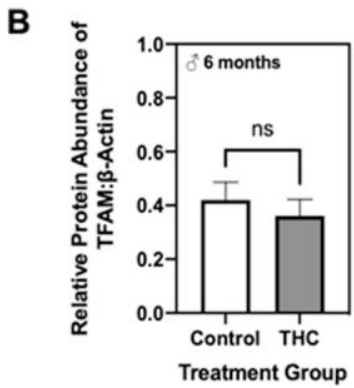

C
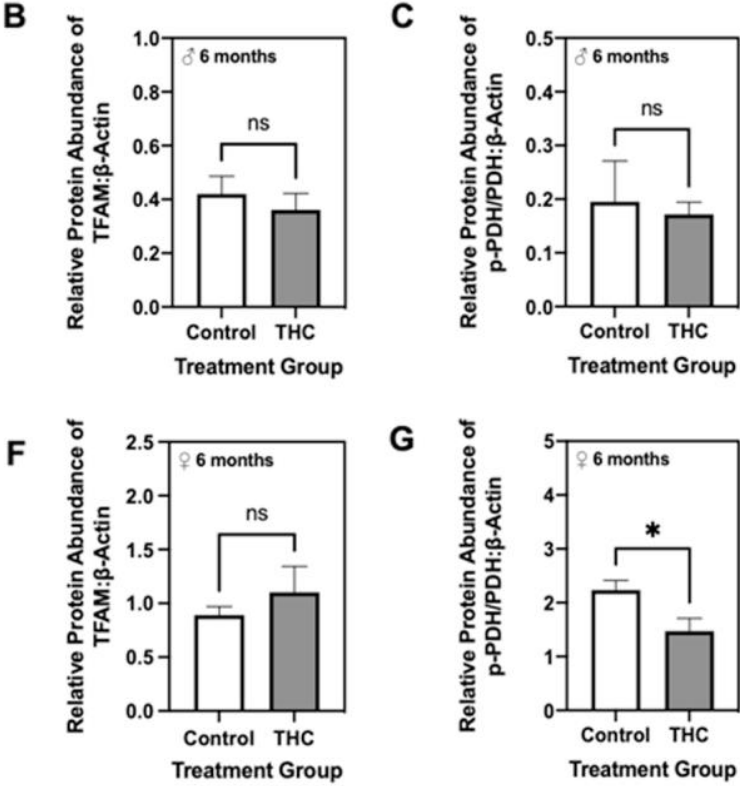

$\mathbf{G}$

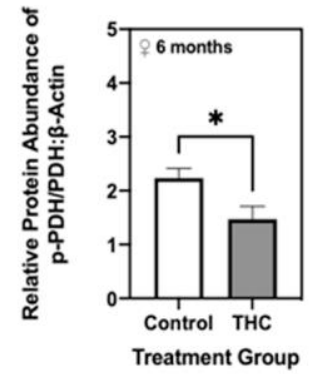

D

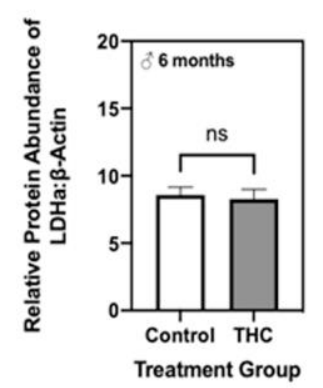

H

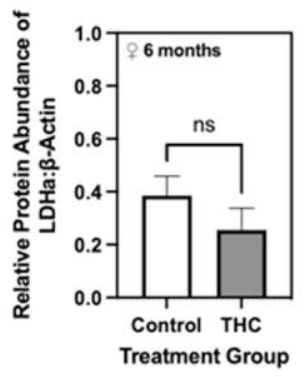

$\mathbf{E}$

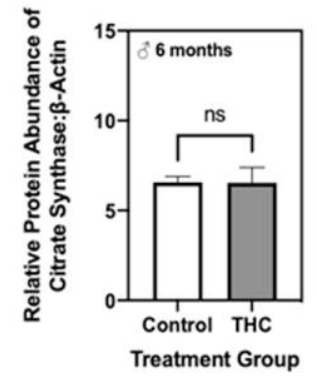

I

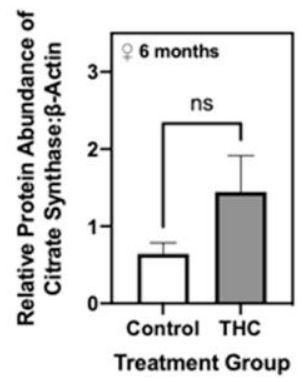

Figure 4. Gestational exposure to $\Delta 9$-tetrahydrocannabinol ( $\Delta 9$-THC) does not impair aerobic metabolism in male or female offspring at six months of age. (A) Representative western immunoblots illustrating hepatic expression of mitochondrial transcription factor A (TFAM), phosphorylated pyruvate dehydrogenase (PDH) to total PDH, lactate dehydrogenase subunit A (LDHa), and citrate synthase in male and female offspring at six months of age. Protein abundances of each enzyme for male offspring (B-E) and female offspring ( $\mathrm{F}-\mathrm{I})$ were normalized to $\beta$-Actin $\pm \mathrm{SEM}$ ( $\mathrm{n}=7-8$ /group). All protein abundances were analyzed using a two-tailed unpaired Student's t-test. *Significant difference $(p<0.05)$.

these same offspring. As expected, transcript abundance of miR-203a-3p was significantly decreased in the livers of adult male offspring with gestational exposure to $\triangle 9$-THC (Figure 9A; $p<0.001)$. Furthermore, livers from three-week old male $\Delta 9$-THC-exposed offspring displayed a trending increase in miR-203a-3p transcript abundance, but this change was not significant (Figure 9E). Similiar to miR-203a-3p, hepatic transcript abundances of miR-29a/b/c was also decreased in sixmonth old male offspring exposed to gestational $\triangle 9$-THC (Figures 9B-D), while the expression of each isoform was unchanged at three weeks of age (Figure 9F-H).

\section{Discussion}

Impairments in the hepatic lipogenic pathway promote excessive production and storage of intracellular triglycerides, leading to the development of obesity and the metabolic syndrome [22]. In the current study, we demonstrate that gestational exposure to $\Delta 9$-THC leads to increased adipose to bodyweight ratio at six months of age, along with elevated hepatic triglyceride levels in adult male offspring. The observed dyslipidemia coincides with increased hepatic expression of lipogenic enzymes at three weeks and six months of age, culminating in accelerated de novo lipogenesis during adult life. Our results suggest that these changes in lipid metabolism occur in a sex-specific manner, as hepatic triglyceride levels were unchanged in adult female offspring exposed to gestational $\Delta 9$ THC. Livers taken from three-week old $\Delta 9$-THC-exposed male offspring also showed increased $4 \mathrm{HNE}$ abundance, suggesting that hepatic lipid peroxidation and oxidative stress first occurs in early 

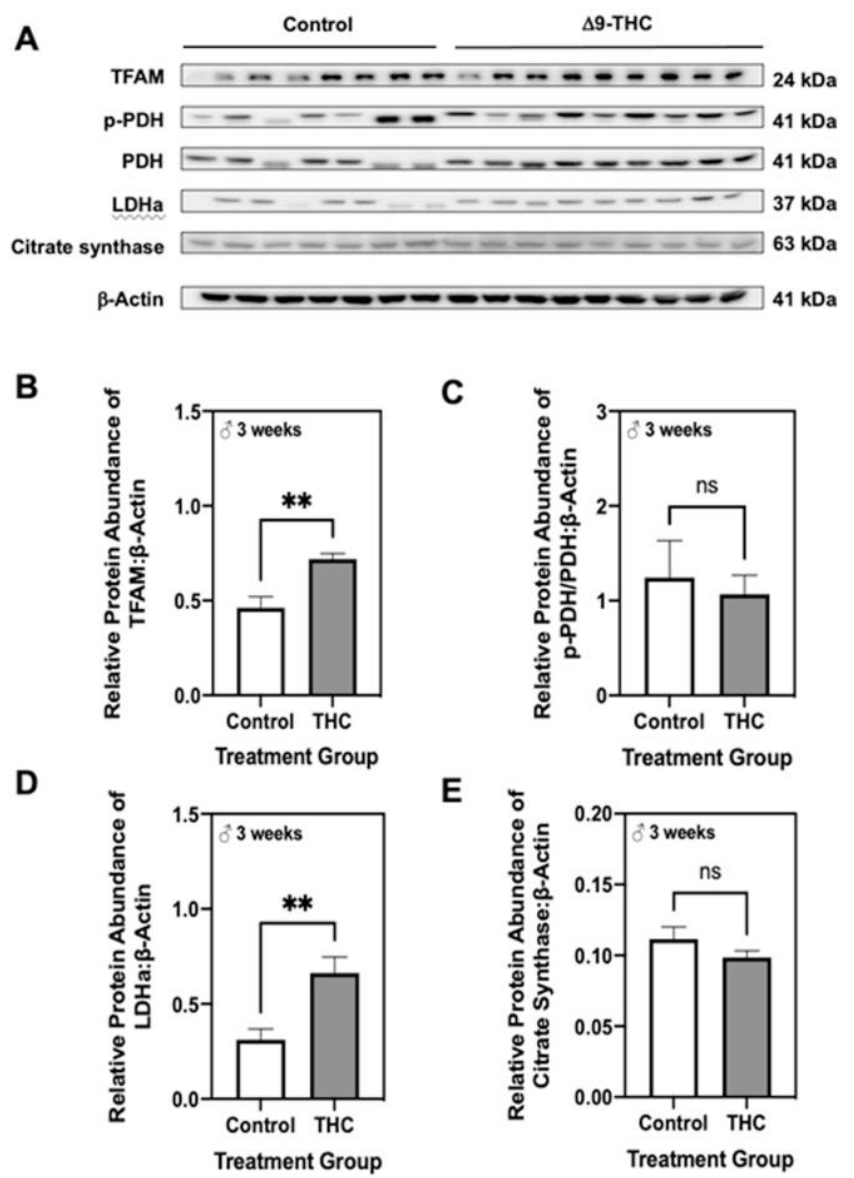

Figure 5. Three-week old male offspring exposed to $\Delta$ 9-tetrahydrocannabinol ( $\Delta 9$-THC) in utero have increased mitochondrial transcription factor A (TFAM) and lactate dehydrogenase subunit A (LDHa). (A) Representative western immunoblots illustrating hepatic expression of mitochondrial transcription factor A (TFAM), phosphorylated pyruvate dehydrogenase (PDH) to total PDH, lactate dehydrogenase subunit A (LDHa), and citrate synthase in male and female offspring at six months of age. Protein abund ances of (B) TFAM, (C) p-PDH[Ser232]/PDH, (D) LDHa and (E) citrate synthase were normalized to $\beta$-Actin \pm SEM ( $\mathrm{n}=7-8 /$ group). All protein abundances were analyzed using a two-tailed unpaired Student's t-test. ${ }^{* *}$ Significant difference $(p<0.01)$.

life. Furthermore, oxidative stress and mitochondrial dysfunction appear to persist into adulthood when hepatic triglycerides are increased. Lastly, our study provides insight into the epigenetic mechanisms that may underly hepatic dyslipidemia in growth-restricted offspring, as adult male offspring exposed to gestational $\Delta 9$-THC exhibited decreased expression of miR-203a-3p and miR$29 \mathrm{a} / \mathrm{b} / \mathrm{c}$, all involved in mitochondrial homeostasis in the liver.

As mentioned previously, there is an inverse relationship between birth weight and long-term metabolic health. We have reported that gestational exposure to $\triangle 9$-THC leads to symmetrical IUGR in rodent offspring, followed by whole body and hepatic catch-up growth by three weeks of age [21]. The occurrence of catch-up growth, or rapid postnatal weight gain, is believed to further exacerbate the risk for metabolic diseases such as obesity, as seen in birth cohorts studying growth-restricted infants from South Africa, Brazil, and the Unites States [57-59]. Here we show that $\Delta 9$-THC-exposed offspring display increased visceral adipose to body weight ratio at six months of age, indicating the development of obesity in adult life. This is consistent with our previous studies of rodent offspring born from models of maternal nicotine exposure and maternal protein restriction, whereby visceral obesity was observed in adult offspring following postnatal catch-up growth [60,61]. Clinically, obese individuals exhibit hypertriglyceridemia in the liver and plasma, often culminating in hepatic steatosis and later NAFLD [62]. In our model, gestational exposure to $\Delta 9-T H C$ led to an increase in 
A
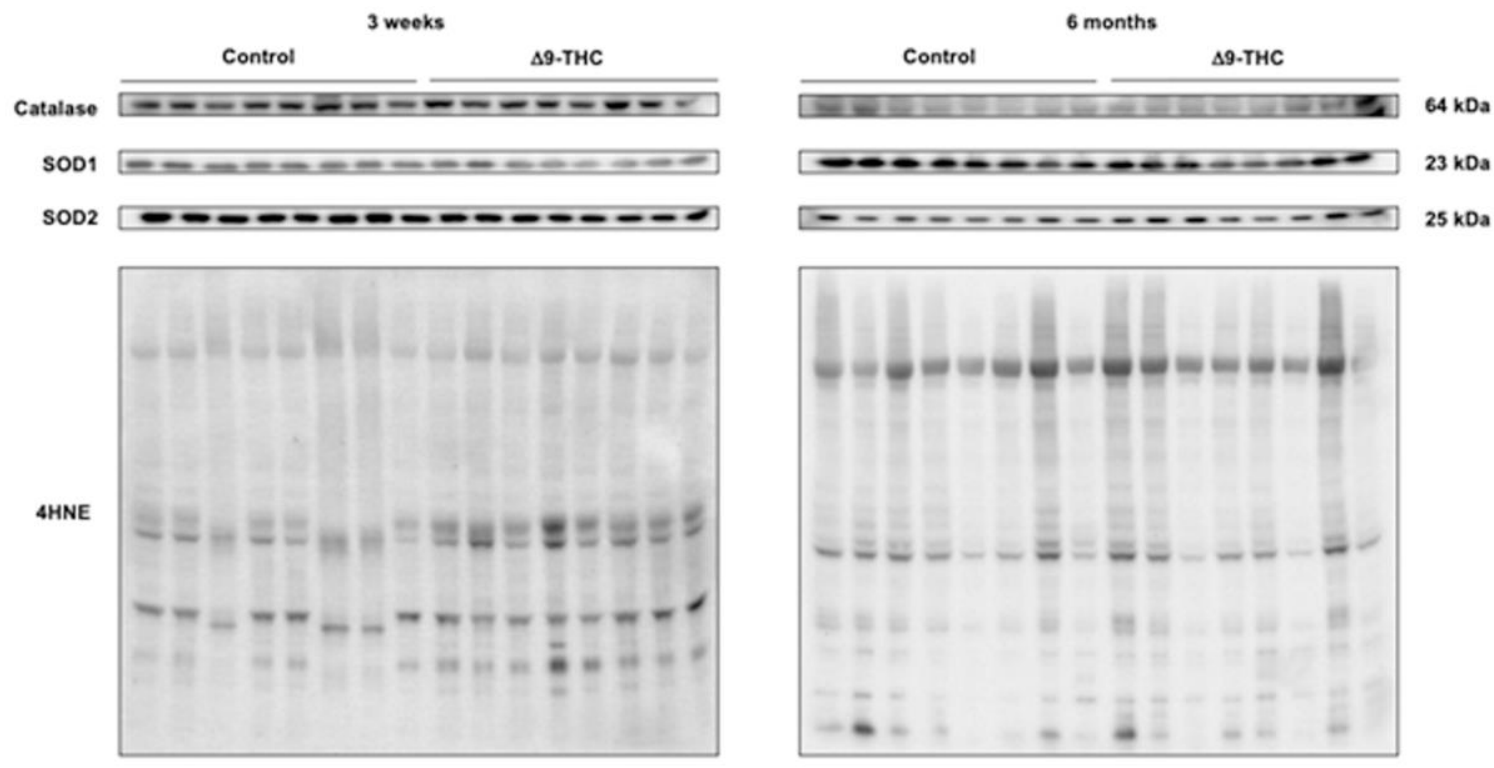

B-Actin
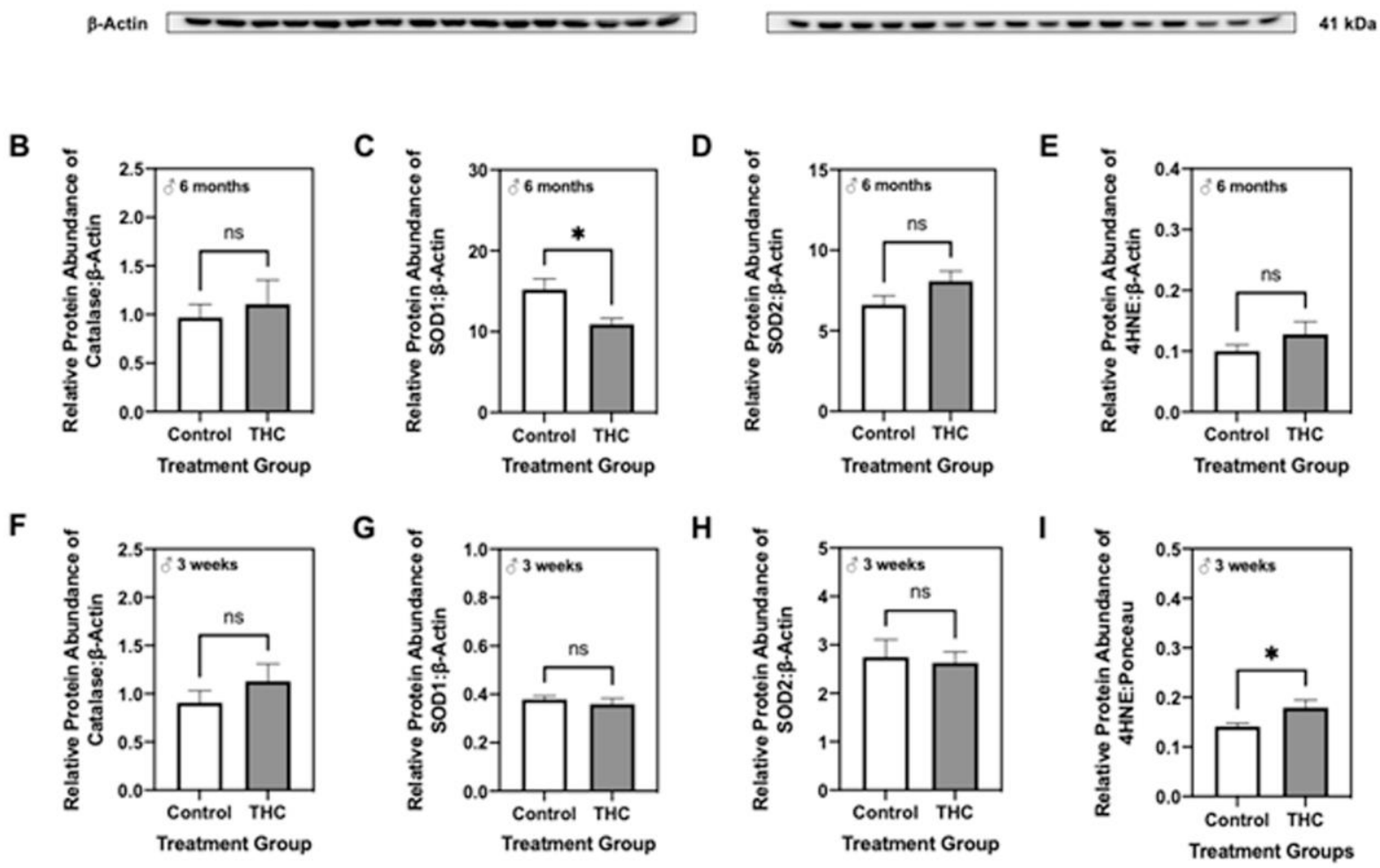

Figure 6. Gestational exposure to $\Delta 9$-tetrahydrocannabinol $(\Delta 9-\mathrm{THC})$ results in hepatic oxidative stress within male offspring at three weeks and six months of age. (A) Representative western immunoblots illustrating hepatic expression of catalase, superoxide dismutase (SOD) 1, SOD2 and 4-hydroxynonenol (4HNE) in male offspring at three weeks and six months. Protein abundance of catalase $(B, F)$, SOD1 $(C, G)$, and SOD2 $(D, H)$ were normalized to $\beta$-Actin \pm SEM (n=7-8/group), while 4HNE abundance (E, I) was expressed as means normalized to total protein abundance \pm SEM $(n=7-8 /$ group $)$. All protein abundances were analyzed using a twotailed unpaired Student's t-test. *Significant difference $(p<0.05)$.

hepatic triglycerides exclusively in male offspring at six months of age. Interestingly, there were no differences in the levels of circulating triglycerides among male offspring, while circulating and hepatic cholesterol levels of both sexes were also unaffected. We have previously reported that female $\triangle 9$-THC exposed offspring exposed to gestational $\triangle 9$-THC do not exhibit differences in circulating estrogen or testosterone relative to control female offspring, so this protective effect cannot be attributed to changes in the circulating levels of androgenic hormones [63]. However, the differences in steroid receptor (i.e., androgen receptor/estrogen receptor) signaling in the liver might 

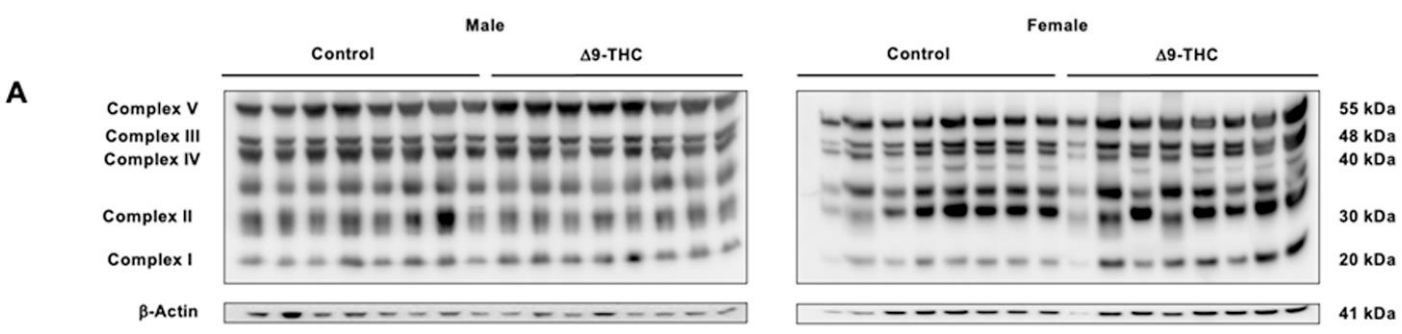

B

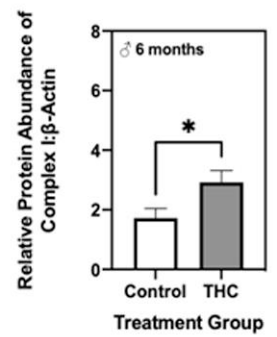

G

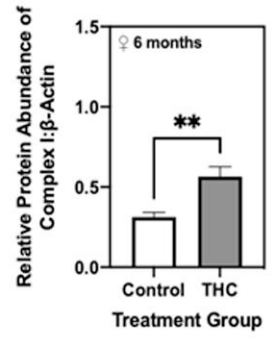

C

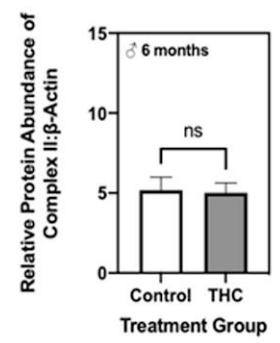

H

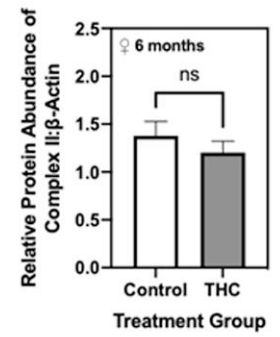

D
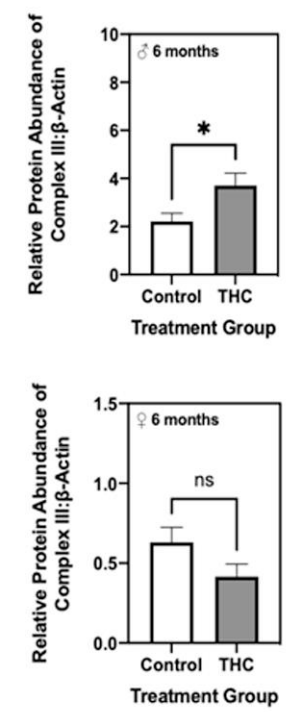

E

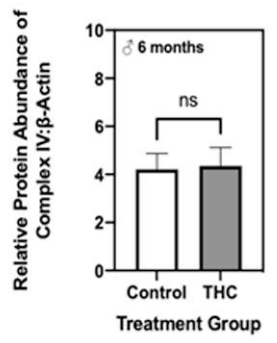

$\mathrm{J}$

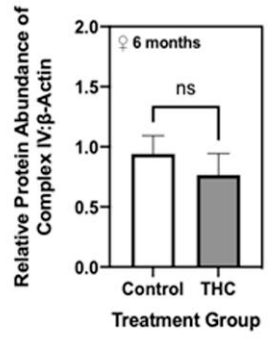

$\mathbf{F}$

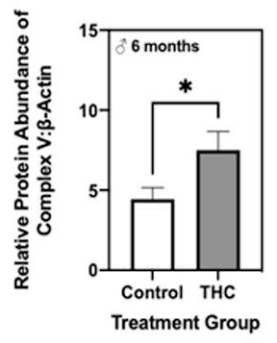

$\mathrm{K}$

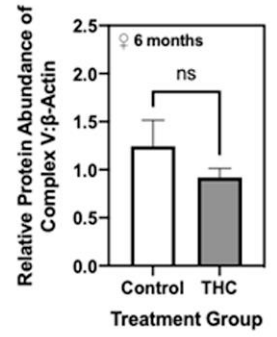

Figure 7. Gestational exposure to $\Delta 9$-tetrahydrocannabinol ( $\Delta 9$-THC) leads to increased complexes I, III and V of the electron transport chain in male offspring at six months of age. (A) Representative western immunoblots illustrating hepatic expression of complexes $\mathrm{I}-\mathrm{V}$ of the electron transport chain in male and female offspring at six months of age. Protein abundances of each enzyme for male offspring (B-F) and female offspring (G-K) were normalized to $\beta$-Actin \pm SEM ( $n=7-8$ /group). All protein abundances were analyzed using a two-tailed unpaired Student's t-test. ${ }^{*}$ Significant difference $(p<0.05),{ }^{* *}$ significant difference $(p<0.01)$.

be involved [64,65]. Overall, there is great evidence to suggest that men are more susceptible than women to the development of hepatic steatosis and NAFLD [66-70]. Studies of healthy men and women demonstrate that men exhibit higher rates of de novo lipogenesis and decreased dietary fatty acid oxidation; therefore, the sexual dimorphism observed among $\Delta 9$-THC-exposed offspring may be attributed to changes in lipogenesis and lipolysis [71,72].

Given the sex-specific differences in hepatic triglyceride content of $\Delta 9$-THC-exposed offspring, we next investigated the relative protein abundance of lipogenic enzymes among male and female adult offspring. At six months of age, male offspring exposed to gestational $\Delta 9$-THC exclusively displayed increased levels of both DGAT1 and DGAT2, along with an increase in the mitochondrial adaptor protein p66Shc. Deletion of DGAT and p66Shc are both independently associated with reduced intracellular accumulation of triglycerides [47,73]; therefore, the upregulation of both DGAT1/2 and p66Shc in the current study likely mediates the increase in hepatic triglycerides of male $\triangle 9$-THC exposed offspring. While adult female $\Delta 9$-THC exposed offspring demonstrated increases in DGAT2, this may not be sufficient in promoting increased de novo lipogenesis in the liver. Instead, a synergistic interaction of multiple enzymes is necessary to elicit changes in lipid metabolism. Since we observed lipogenic changes in male offspring exposed to gestational $\Delta 9-\mathrm{THC}$ in adulthood, we further assessed if this occurred earlier (e.g., three weeks) coinciding with the completion of hepatic catch-up growth. $\triangle 9$-THC exposed male offspring exposed exhibited increased abundance of ACC $\alpha$, SCD, FABP1 and DGAT2 at three weeks of age, suggesting that catch-up growth may instigate de novo lipogenesis. Incidentally, recent studies have determined that FABP1 is an important regulator of $\triangle 9$-THC hepatic transport and biotransformation [74,75]. Many hepatic pathophysiologies, including NAFLD, are associated with increased expression of FABP1, while its knockdown reduces hepatic triglyceride accumulation and lipid peroxidation [76]. This is consistent with our current study, as three-week old male offspring displayed increased abundance of $4 \mathrm{HNE}$ (i.e., a marker of lipid peroxidation) concomitant with elevated FABP1. Since FABP1 is involved in 


\section{A}

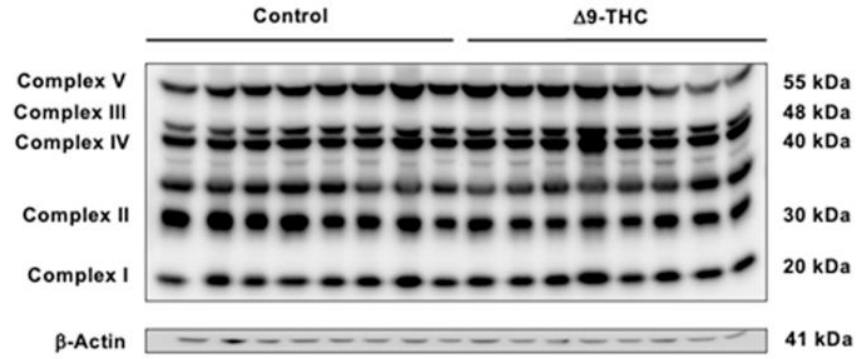

B

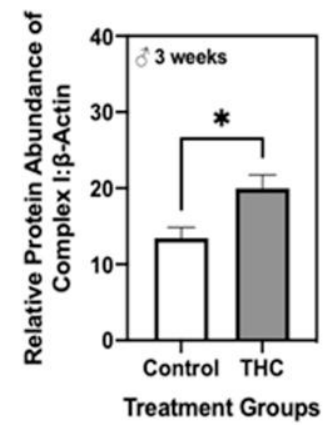

E

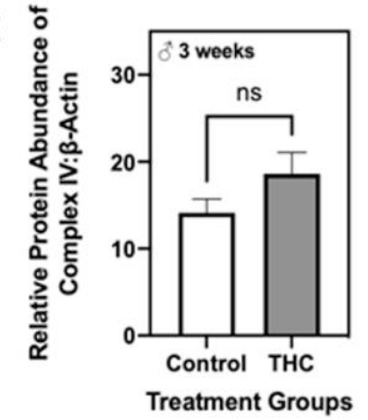

C :

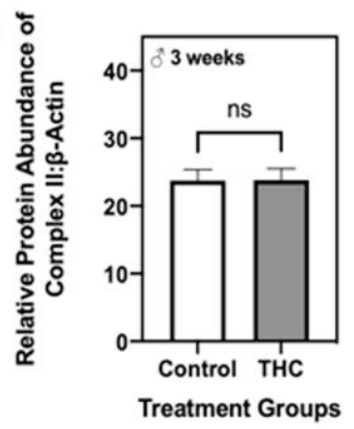

$\mathbf{F}$

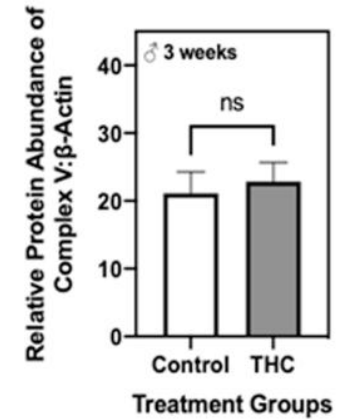

D :

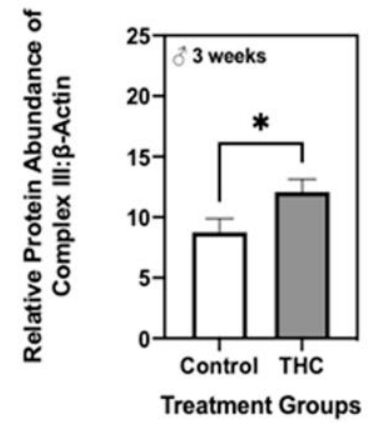

Figure 8. Gestational exposure to $\Delta$-tetrahydrocannabinol ( $\Delta 9$-THC) leads to increased complexes I and III of the electron transport chain in male offspring at three weeks of age. (A) Representative western immunoblots illustrating hepatic expression of complexes I-V of the electron transport chain in male offspring at three weeks of age. Protein abundances of complex I (B), II (C), III (D), IV (E) and V (F) were normalized to $\beta$-Actin \pm SEM ( $n=7-8$ /group). All protein abundances were analyzed using a two-tailed unpaired Student's t-test. *Significant difference $(p<0.05)$.

$\triangle 9$-THC metabolism, it is possible that these offspring have altered expression of FABP1 in response to exposure to $\triangle 9$-THC in utero. Mice with knockdown of hepatic FABP1 also exhibit decreased expression of DGAT1 and DGAT2 in the liver[76]; therefore, early elevation of FABP1 in $\triangle 9$-THCexposed male offspring could be involved in the upregulation of DGAT and de novo lipogenesis later in life.

Oxidative stress and mitochondrial dysfunction are highly prevalent among metabolic diseases involving the liver. Elevated reactive oxygen species (ROS) and mitochondrial abnormalities are implicated in the pathogenesis of NAFLD; however, the mechanisms by which this occurs are not completely understood [77-80]. In vitro studies have established that ROS are detrimental to hepatic lipid metabolism and mitochondrial function, as treatment of HepG2 cells and primary hepatocytes with hydrogen peroxide promotes the accumulation of triglycerides and cholesterol [81]. Additionally, lipid-induced elevation of ROS has been shown to impair mitochondrial function in the human hepatoblastoma C3A cell line, along with increased gluconeogenesis and ketogenesis [82]. Numerous animal models have also found that IUGR offspring exhibit indices of hepatic oxidative stress (e.g., increased lipid peroxidation and ROS; altered expression and activity of antioxidant enzymes) and impaired mitochondrial function (e.g., aberrant expression and activity of pyruvate dehydrogenase, citrate synthase, and complexes of the electron 
A

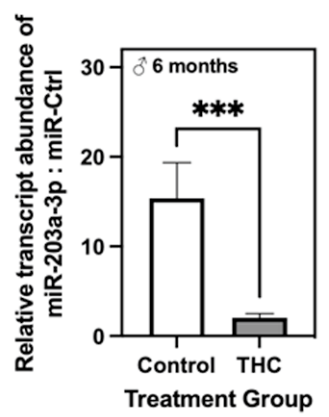

E

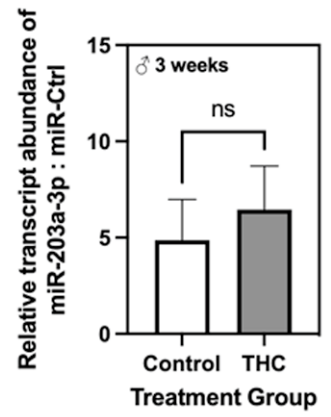

B

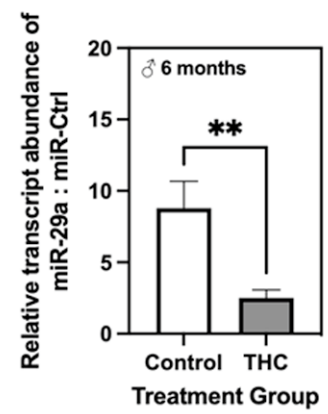

$\mathbf{F}$

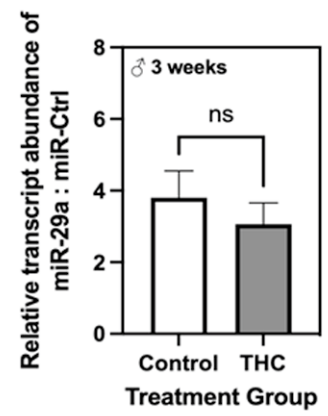

C

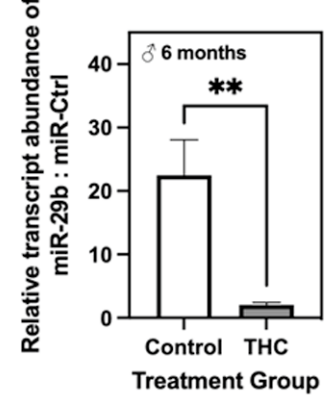

$\mathbf{G}$

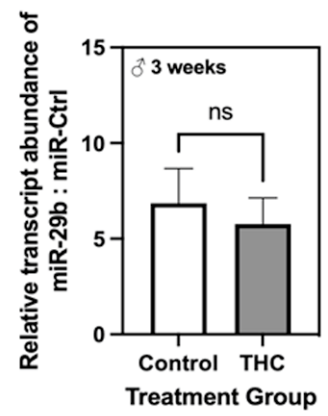

D

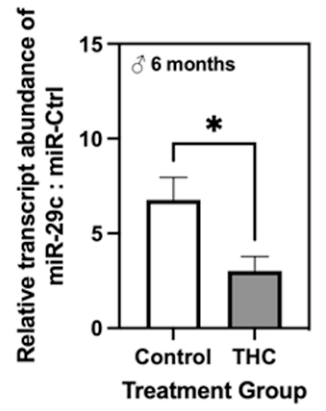

H

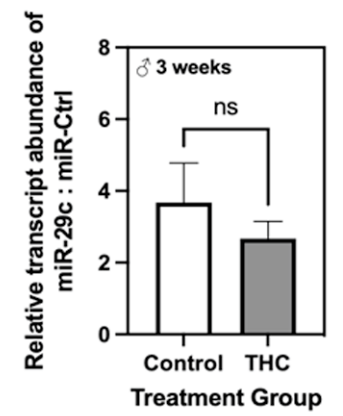

Figure 9. Quantitative RT-PCR analysis of miR-203a-2p and miR-29a/b/c in the livers of male offspring at six months (A-D) and three weeks $(\mathrm{E}-\mathrm{H})$ following gestational exposure to $\Delta$ 9-tetrahydrocannabinol ( $\Delta 9$-THC). Relative amounts of all miRs were normalized to that of miRNA standard control. Data are expressed as means \pm SEM ( $n=7-8 /$ group). Groups at each time point were compared using Student's two-tailed unpaired t-test. ${ }^{*}$ Significant difference $(p<0.05),{ }^{* *}$ significant difference $(p<0.01),{ }^{* *}$ significant difference $(p<0.001)$

transport chain; disrupted ATP synthesis) during neonatal and adult life [40-43,45]. In particular, we have shown that hepatic p66Shc is elevated in adult male offspring subject to maternal protein restriction attributed to postnatal catch-up growth [45]. P66Shc is a key regulator of cellular redox state, lifespan, and mitochondrial metabolism, as it interacts with cytochrome $\mathrm{C}$ under conditions of cell stress to stimulate the production of ROS [46]. This mitochondrial-induced oxidative stress often leads to apoptosis, cellular senescence and compromised aerobic metabolism [46,83-85], making p66Shc an attractive target in mitigating metabolic disease. Again, studies have also postulated that p66Shc is involved in the accumulation of intracellular lipids [47]. Here we found that adult male offspring exposed to gestational $\triangle 9$-THC have increased protein abundance of p66Shc in the liver, concomitant with decreased abundance of SOD1. While we did not directly measure hepatic ROS, together these results suggest that oxidative stress occurs in the liver following exposure to gestational $\triangle 9$-THC and catch-up growth. To investigate this further, we assessed the levels of proteins involved in mitochondrial function and aerobic metabolism (i.e., TFAM, p-PDH[Ser232], LDHa, citrate synthase, and complexes of the electron transport chain). While the expression of TFAM, p-PDH[Ser232], LDHa and citrate synthase was unchanged in adult male offspring, they did have increased amounts of complexes I, III and V. This is noteworthy as increases in complexes I and III are associated with elevated superoxide production via increased flow of electrons through the subunits of these enzymes [55]. These data, in combination with our p66Shc and SOD1 findings, further support the idea that these offspring exhibit hepatic oxidative stress and altered oxidative phosphorylation. Complexes I and III were also found to be increased in three-week old male offspring, suggesting that oxidative stress occurs in early life following $\Delta 9$-THC exposure and catchup growth.

To date, little is known about the epigenetic mechanisms underlying metabolic diseases in IUGR offspring. One such mechanism is miRs, which are small, non-coding RNA molecules that silence target genes through mRNA degradation or repressed protein translation. Numerous miRs have been demonstrated to have aberrant postnatal expression in growth-restricted offspring, leading to cellular stress that precedes impaired function of metabolic organs [51,86-88]. Recent studies have found that the translation of p66Shc protein is directly inhibited by miR-203a-3p, leading to the attenuation of liver injury and fibrosis in mice [56]. Here we observed robust decreases in the expression of miR-203a-3p in the livers of male offspring exposed to gestational $\Delta 9-\mathrm{THC}$, but 
only at six months of age. This is consistent with the observed increase in p66Shc protein levels at this time point, indicating that miR-203a-3p may be implicated in the development of oxidative stress and de novo lipogenesis in these offspring. We further quantified the expression of miR-29, as we have found this miR family to be altered in the livers of adult male protein-restricted offspring following catch-up growth [51]. Similar to our findings of miR-203a-3p, adult male $\Delta$ 9-THC-exposed offspring exhibited decreased hepatic expression of all three isoforms of miR-29 at six months of age. This is of great interest as miR-29 expression is downregulated in patients with advanced liver fibrosis and cirrhosis [89]. Conversely, when present at high levels, miR-29 can alleviate hepatocellular steatohepatitis, fibrosis and cirrhosis in mice [89-91]. Collectively, given that each of these miRs were down-regulated exclusively during adult life, it is conceivable that early catchup growth of the liver culminates in long-term oxidative stress and impaired hepatic lipid metabolism through epigenetic regulation of gene expression. Future in vitro studies are warranted to investigate this relationship further in efforts to reveal additional metabolic targets that become dysregulated with altered expression of $\mathrm{miR}-203 \mathrm{a}-3 \mathrm{p}$ and $\mathrm{miR}-29 \mathrm{a} / \mathrm{b} / \mathrm{c}$ in the liver.

In summary, this study demonstrates for the first time that gestational exposure to $\Delta 9-\mathrm{THC}$ leads to hepatic dyslipidemia in adult male offspring. We postulate that this occurs as a result of accelerated triglyceride synthesis (i.e., de novo lipogenesis) in the liver, as multiple lipogenic enzymes are elevated at both three weeks and six months of age. Given that FABP1 is involved in the metabolism and transport of $\triangle 9-\mathrm{THC}$, it is possible that gestational exposure to $\Delta 9-\mathrm{THC}$ in combination with catch-up growth leads to early elevation of FABP1 in the livers of male offspring. This may further contribute to the observed increase in lipid peroxidation and lipogenic enzyme expression in male offspring at three weeks of age. Hepatic lipid overload is known to further increase oxidative stress and mitochondrial dysfunction; therefore, this process may occur in a cyclical manner as indicated in Figure 10. Altered expression of miRs may also contribute to these molecular changes, as we have indicated that miR-203a-3p and miR-29a/b/c are downregulated in response to $\triangle 9$-THC exposure and catch-up growth. Additional long-term studies will be important in determining if this later culminates in hepatic pathologies such as NAFLD and cirrhosis of the liver. While much remains unknown about the role of prenatal cannabinoids on offspring outcomes, it is clear that long-term metabolic health becomes compromised as a result. We have previously shown that exposure to $\triangle 9$-THC specifically has effect on glucose tolerance and cardiovascular function $[63,92]$, and our current study demonstrates that it further contributes to dyslipidemia. Overall, these data provide great insight into the effects of gestational $\triangle 9$-THC exposure on the development and function of the liver, as well as the fundamental molecular mechanisms that underlie the metabolic dysfunction of growth-restricted offspring.

\section{Materials and Methods}

\subsection{Animals and experimental handling}

All procedures were performed according to guidelines set by the Canadian Council of Animal Care, and Animal Use Protocol (AUP \#2019-126) was approved by the Animal Care Committee at The University of Western Ontario. All investigators understood and followed the ethical principles outlined by Grundy [93], and study design was informed by ARRIVE guidelines [94]. Pregnant female Wistar rats were purchased from Charles River (La Salle, St. Constant QC). Dams arrived at the animal care facility at gestational day 3 (GD3) and were left to acclimatize to environmental conditions for three days. All animals were maintained at $22{ }^{\circ} \mathrm{C}$ on a $12: 12 \mathrm{~h}$ lightdark cycle in the animal care facility, while food and water were provided ad libitum for the entire duration of the experimental protocol. Dams were randomly assigned to receive daily intraperitoneal (i.p.) injection of either vehicular control (1:18 cremophor:saline) or $3 \mathrm{mg} / \mathrm{kg} \Delta 9-\mathrm{THC}$ (Sigma-Aldrich, St. Louis) from E6.5 to E22 ( $n=14$, where litter is the statistical unit) as previously performed [21]. This dose of $\triangle 9$-THC was selected as it results in rodent plasma concentrations (8.6$12.4 \mathrm{ng} / \mathrm{mL}$ ) that are reflective of those found in human recreational cannabis smokers (using $6 \% \Delta 9$ THC) $0-22 \mathrm{~h}$ post-inhalation $(13-63 \mathrm{ng} / \mathrm{mL})$, as well as in the aborted fetal tissues of pregnant cannabis users (4-287 ng/mL)[95-97]. This dose and method of delivery has also been demonstrated to have no impact on maternal or litter outcomes in rats [21,98,99]. An oral route of administration was not chosen as to avoid the poor bioavailability and slowed adsorption of $\Delta 9$-THC when ingested 


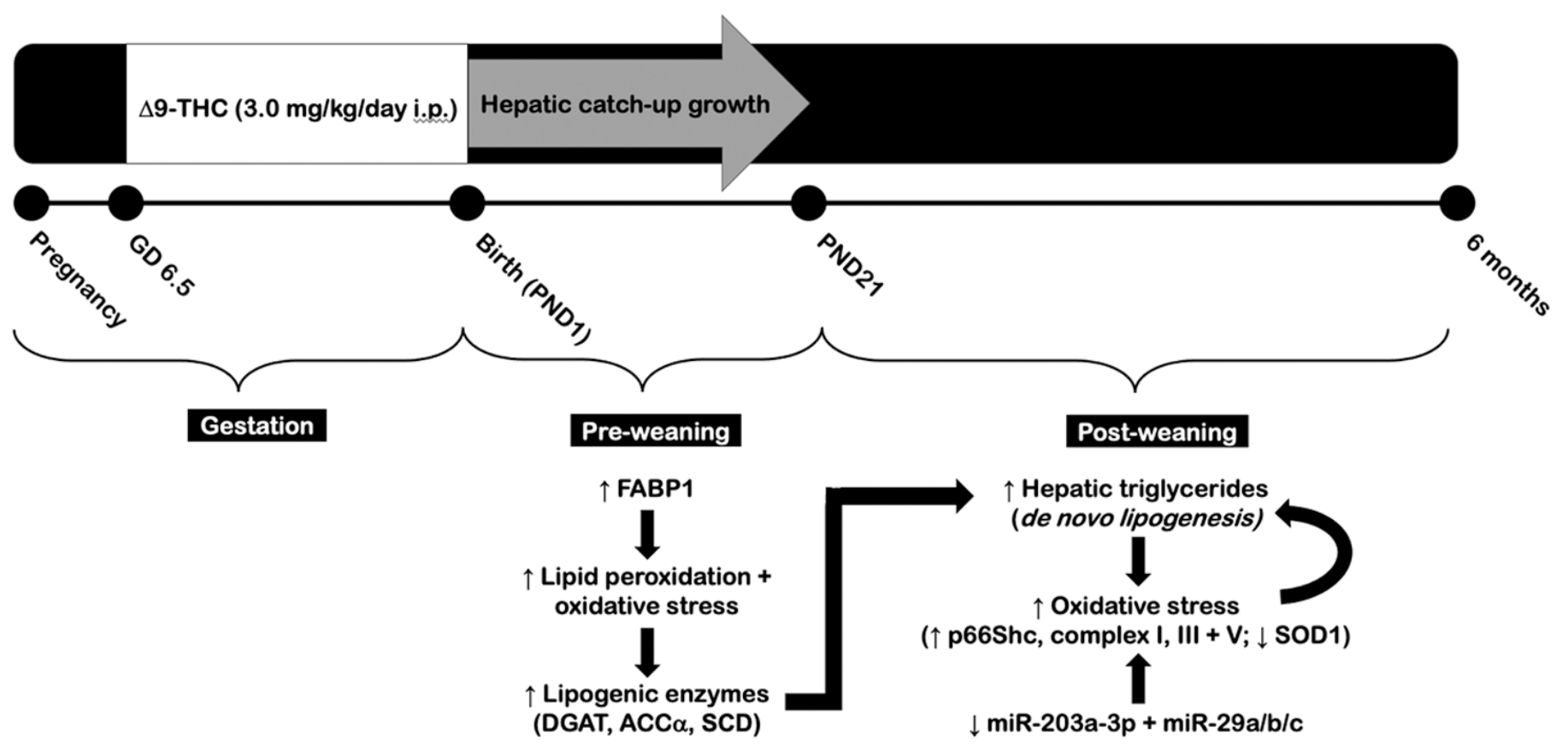

Figure 10. Proposed schematic illustrating the effects of gestational exposure to $\Delta$-tetrahydrocannabinol ( $\Delta 9$-THC) on the male rat liver. In summary, in utero exposure to $\Delta 9$-THC led to symmetrical intrauterine growth restriction in the affected offspring, followed by hepatic catch-up growth and increased visceral adiposity during adult life. Male offspring exposed to gestational $\Delta 9$-THC exhibited increased FABP1 and oxidative stress at three weeks of age, leading to increased de novo lipogenesis at six months. Adult male offspring also demonstrated oxidative stress and mitochondrial dysfunction, which may occur as a result of elevated hepatic triglyceride content. The presence of oxidative stress and mitochondrial dysfunction in combination with downregulated miR-203a$3 \mathrm{p}$ and $\mathrm{miR}-29 \mathrm{a} / \mathrm{b} / \mathrm{c}$ may further promote impaired lipid metabolism of the liver in a positive-feedback manner.

with food, and because edibles are the least popular route of administration of cannabis among pregnant women $[4,100]$. Injections were initiated at E6.5 because $\Delta 9$-THC can interfere with implantation of the blastocyst and induce spontaneous abortion [100].

Maternal food intake and body weight were monitored daily throughout the entire gestational period. Dams were allowed to deliver normally, and all pups were weighed at birth. Litters were randomly culled to eight offspring per litter to ensure uniformity of litter size between treatment groups. As previously reported, we and others have found the selected dose of $\triangle 9$-THC to have no impact on maternal or litter outcomes $[20,21,98,99]$. Liver weights of culled offspring were recorded and compared to body weight as a measure of fetal growth restriction and postnatal catch-up growth [21]. The food intake of these offspring was monitored by measuring their daily food consumption from PND50-60, as previously published [51]. The remaining offspring were fasted for 24 hours before being euthanized via i.p. injection of $100 \mathrm{mg} / \mathrm{kg}$ pentobarbital at either postnatal day (PND) 21 or six months of age ( $\mathrm{n}=8$ males/ 8 females per group), followed by necropsy to examine the effects of $\triangle 9$-THC on metabolic and molecular outcomes. The right medial hepatic lobe was collected and immediately flash-frozen in liquid nitrogen, followed by storage at $-80{ }^{\circ} \mathrm{C}$ until further use. Visceral fat was also weighed and compared to body weight at six months as a measure of obesity. Blood was also collected, centrifuged, and stored at $-80^{\circ} \mathrm{C}$.

\subsection{Hepatic triglyceride measurements}

Circulating and hepatic triglyceride and cholesterol measurements were detected using the Cobas ${ }^{\circledR}$ Mira S analyzer as previously published [30]. For triglyceride measurements, triglycerides were hydrolyzed by lipoprotein lipase to glycerol and fatty acids. Glycerol was then phosphorylated to glycerol-3-phosphate by ATP in a reaction catalyzed by glycerol kinase (GK). The oxidation of glycerol-3-phosphate was catalyzed by glycerol phosphate oxidase (GPO) to form dihydroxyacetone phosphate and hydrogen peroxide $\left(\mathrm{H}_{2} \mathrm{O}_{2}\right)$. In the presence of peroxidase, $\mathrm{H}_{2} \mathrm{O}_{2}$ alters the oxidative coupling of 4-chlorophenol and 4-aminophenazone to form a red-colored quinoneimine dye, which was measured at $512 \mathrm{~nm}$. The increase in absorbance is directly proportional to the concentration of 
triglycerides in the sample. For cholesterol measurements, cholesterol esterase cleaved cholesterol esters, which then were converted to choleste-4-en-3-one and $\mathrm{H}_{2} \mathrm{O}_{2}$ by cholesterol oxidase. Cholesterol levels were quantified using a colorimetric assay that measured the breakdown of $\mathrm{H}_{2} \mathrm{O}_{2}$ via the Trinder reaction as previously described [31].

\section{3. $R N A$ isolation and quantitative real-time PCR analysis}

MicroRNAs were isolated from frozen liver samples using a miRNeasy kit (QIAGEN Canada), followed by spectrophotometric analysis with a Nanodrop 2000. $0.5 \mu \mathrm{g}$ of each miRNA sample was then reverse transcribed into cDNA using a miScript II RT kit (QIAGEN Canada) and stored at $20{ }^{\circ} \mathrm{C}$. Forward sequence primers for miR-203a-3p, miR-29a, miR-29b, and miR-29c were purchased (QIAGEN Canada), while a universal reverse sequence primer for miRNAs was used as part of a miScript SYBR green PCR kit (QIAGEN Canada). MiRNA transcripts were amplified via quantitative real-time PCR (qRT-PCR) using the Bio-Rad CFX384 Real Time System, using cycling conditions as previously published[51]. Cq values obtained for miRs of interest were normalized to that of a miRNA standard control (Ctrl_miRTC_1; QIAGEN Canada), and relative transcript abundance was calculated for each target using the comparative $\Delta \mathrm{Ct}$ method.

\subsection{Protein extraction and western immunoblot}

Whole-cell protein lysates were isolated using the protocol previously described by Barra et al. [52] Prior to western immunoblotting, loading samples were prepared using sample lysates, NuPAGE reducing agent (10X; Invitrogen), NuPAGE LDS sample buffer (4X; Invitrogen), and deionized water. Loading samples were heated at $70{ }^{\circ} \mathrm{C}$ to denature proteins, while a separate set of loading mixes were kept unheated for mitochondrial OXPHOS immunoblots. Samples were loaded into wells of $4-12 \%$ Bis-Tris gels (Invitrogen) at $20-30 \mu \mathrm{g}$ per well ( $\mathrm{n}=8 /$ group), followed by separation via gel electrophoresis. Proteins were transferred onto PVDF membranes (Thermo Scientific) at 75V for two hours, followed by one hour of blocking in 1X Tris-buffered saline/Tween20 (TBST) buffer with either 5\% non-fat milk (Carnation) or 3\% bovine serum albumin (BSA; Fisher). Membranes were probed with primary antibodies overnight, followed by probing with secondary antibodies for one hour (Table 2). Immunoreactive bands were visualized using BioRad Clarity Max Western ECL Substrate solution and imaged using a BioRad ChemiDoc XRS+ Imaging System. Resulting bands were analyzed using BioRad Image Lab ${ }^{\mathrm{TM}}$ Software. Band intensities of target proteins were normalized to $\beta$-Actin as previously published [45], with the exception of 4hydroxynonenol which was normalized to total protein content detected with Ponceau staining [45,101].

\subsection{Statistical analyses}

At each timepoint, the selected offspring were taken from separate litters to avoid litter bias (i.e., $\mathrm{n}=1$ represents pups from a single dam), where a sample size of $\mathrm{n}=7-8$ was used for each sex per group. This sample size was chosen based on our previous studies to achieve a statistically significant difference with an expected standard deviation of $15 \%$ or less $[63,102]$. All statistical analyses were performed using GraphPad Prism 9 software. Results were expressed as means of normalized values \pm SEM, and the threshold for significance was set as $p<0.05$. Organ and body weight data were analyzed using Student's two-tailed unpaired t-test when examining both sexes together (e.g., PND1), while sex-specific effects were analyzed by two-way ANOVA followed by a Holm-Sidak-corrected multiple comparisons test. All immunoblot data were analyzed using Student's two-tailed unpaired t-test. Triglyceride and cholesterol measurements obtained from lipid analyses were analyzed by two-way ANOVA, followed by Holm-Sidak-corrected multiple comparisons test between $\triangle 9$-THC offspring and their sex-matched controls. Grubbs' test was utilized to detect any statistical outliers.

Author Contributions: SLO, RP, and DBH were involved in the design, execution and interpretation of experiments and results. KL was involved with implementing the animal model. SRL contributed to the design of animal experiments, as well as the preparation and dosing of

Table 2. Western blot primary and secondary antibodies, dilutions and company/catalogue information. 


\begin{tabular}{|c|c|c|c|}
\hline Antibody Name & Source & Dilution & Company (Catalogue No.) \\
\hline $\mathrm{ACC} \alpha(\mathrm{H}-76)$ & Rabbit polyclonal & $1: 500$ & $\begin{array}{l}\text { Santa Cruz Biotechnology Inc., Santa Cruz, CA, USA (sc- } \\
30212 \text { ) }\end{array}$ \\
\hline FAS (C20G5) & Rabbit monoclonal & 1:1000 & $\begin{array}{l}\text { Cell Signaling Technology Inc., Danvers, MA, USA } \\
(\# 3180)\end{array}$ \\
\hline SCD (H300) & Rabbit polyclonal & $1: 250$ & $\begin{array}{l}\text { Santa Cruz Biotechnology Inc., Santa Cruz, CA, USA (sc- } \\
\text { 30081) }\end{array}$ \\
\hline FABP1 (D2A3X) & Rabbit monoclonal & 1:1000 & $\begin{array}{l}\text { Cell Signaling Technology Inc., Danvers, MA, USA } \\
(\# 13368)\end{array}$ \\
\hline DGAT1 & Rabbit polyclonal & 1:1000 & Novus Biologicals, Centennial, CO, USA (NB110-41487) \\
\hline DGAT2 (4C1) & Mouse monoclonal & 1:1000 & $\begin{array}{l}\text { Santa Cruz Biotechnology Inc., Santa Cruz, CA, USA (sc- } \\
\text { 293211) }\end{array}$ \\
\hline SHC & Mouse monoclonal & 1:1000 & BD BioSciences, San Jose, CA, USA (610879) \\
\hline TFAM (D5C8) & Rabbit monoclonal & 1:1000 & $\begin{array}{l}\text { Cell Signaling Technology Inc., Danvers, MA, USA } \\
(8076)\end{array}$ \\
\hline $\begin{array}{l}\text { pSer(232) pyruvate } \\
\text { dehydrogenase }\end{array}$ & Rabbit polyclonal & 1:1000 & EMD Millipore, Etobicoke, ON, Canada (AP1063) \\
\hline $\begin{array}{l}\text { Pyruvate } \\
\text { dehydrogenase }\end{array}$ & Rabbit polyclonal & 1:1000 & $\begin{array}{l}\text { Cell Signaling Technology Inc., Danvers, MA, USA } \\
(2784)\end{array}$ \\
\hline LDHa & Rabbit polyclonal & 1:1000 & $\begin{array}{l}\text { Cell Signaling Technology Inc., Danvers, MA, USA } \\
(2012)\end{array}$ \\
\hline Citrate Synthase & Rabbit polyclonal & 1:1000 & Provided by Dr. S. Raha, McMaster University \\
\hline Catalase (H-300) & Rabbit polyclonal & 1:1000 & $\begin{array}{l}\text { Santa Cruz Biotechnology Inc., Santa Cruz, CA, USA (sc- } \\
50508 \text { ) }\end{array}$ \\
\hline $\begin{array}{l}\text { Superoxide dismutase } \\
\text { (SOD)-1 (FL-154) }\end{array}$ & Rabbit polyclonal & 1:1000 & $\begin{array}{l}\text { Santa Cruz Biotechnology Inc., Santa Cruz, CA, USA (sc- } \\
\text { 11407) }\end{array}$ \\
\hline $\begin{array}{l}\text { Superoxide dismutase } \\
\text { (SOD)-2 (FL-222) }\end{array}$ & Rabbit polyclonal & 1:1000 & $\begin{array}{l}\text { Santa Cruz Biotechnology Inc., Santa Cruz, CA, USA (sc- } \\
30080 \text { ) }\end{array}$ \\
\hline 4-hydroxynonenal & Mouse monoclonal & 1:1000 & R\&D Systems, Oakville, ON, Canada (MAB3249) \\
\hline rodent & Mouse monoclonal & 1:1000 & Abcam Inc., Toronto, ON, Canada (ab110413) \\
\hline$\beta$-Actin Peroxidase & Mouse monoclonal & $1: 25000$ & Sigma Aldrich Co., St. Louis, MO, USA (A3854-200UL) \\
\hline $\begin{array}{ll}\text { Goat anti-rabbit } & \text { IgG } \\
\text { HRP-linked } & (\mathrm{H}+\mathrm{L} \\
\text { chain) } & \\
\end{array}$ & N/A & 1:10000 & $\begin{array}{l}\text { Cell Signaling Technology Inc., Danvers, MA, USA } \\
\text { (7074P2) }\end{array}$ \\
\hline $\begin{array}{l}\text { Horse anti-mouse } \operatorname{IgG} \\
\text { HRP-linked } \\
\text { chain) }\end{array}$ & N/A & 1:10000 & $\begin{array}{l}\text { Cell Signaling Technology Inc., Danvers, MA, USA } \\
\text { (7076S) }\end{array}$ \\
\hline
\end{tabular}

vehicle and $\triangle 9$-THC in vivo. SLO was responsible for writing the manuscript, while all authors were involved in editing drafts of the manuscript.

Funding: This work was supported by a Canadian Institutes of Health Research Catalyst Grant (CRU1126) to DBH and a Canadian Heart and Stroke Foundation Grant-in-Aid (G-19-0026343) to DBH. An NSERC CGS-D was awarded to SLO.

Institutional Review Board Statement: All procedures were performed according to guidelines set by the Canadian Council of Animal Care, and Animal Use Protocol (AUP \#2019-126) was approved by the Animal Care Committee at The University of Western Ontario. All investigators understood and followed the ethical principles outlined by Grundy, and study design was informed by ARRIVE guidelines.

\section{Data Availability Statement: N/A}


Acknowledgments: The authors thank Dr. D Betts, Dr. T Regnault, and Dr. S Raha for generously sharing their antibodies, as well as Brian Sutherland, Cindy Sawyez and Dr. Nica Borradaile for their assistance with lipid analyses.

Conflicts of Interest: The authors declare no conflict of interest.

\section{References}

1. Canadian Cannabis Survey 2019 - Summary - Canada.Ca.

2. Marijuana Use during Pregnancy and Lactation. Obstetrics and Gynecology 2017, 130, e205-e209.

3. Westfall, R.E.; Janssen, P.A.; Lucas, P.; Capler, R. Survey of Medicinal Cannabis Use among Childbearing Women: Patterns of Its Use in Pregnancy and Retroactive Self-Assessment of Its Efficacy against "Morning Sickness." Complement Ther Clin Pract 2006, 12, 27-33, doi:10.1016/j.ctcp.2005.09.006.

4. Chang, J.C.; Tarr, J.A.; Holland, C.L.; De Genna, N.M.; Richardson, G.A.; Rodriguez, K.L.; Sheeder, J.; Kraemer, K.L.; Day, N.L.; Rubio, D.; et al. Beliefs and Attitudes Regarding Prenatal Marijuana Use: Perspectives of Pregnant Women Who Report Use. Drug Alcohol Depend 2019, 196, 14-20, doi:10.1016/j.drugalcdep.2018.11.028.

5. Brown, R.A.; Dakkak, H.; Gilliland, J.; Seabrook, J.A. Predictors of Drug Use during Pregnancy: The Relative Effects of Socioeconomic, Demographic, and Mental Health Risk Factors. J Neonatal Perinatal Med 2019, 12, 179-187, doi:10.3233/NPM1814 .

6. $\quad$ English, D.R.; Hulse, G.K.; Milne, E.; Holman, C.D.J.; Bower, C.I. Maternal Cannabis Use and Birth Weight: A Meta-Analysis. Addiction 1997, 92, 1553-1560, doi:10.1111/j.1360-0443.1997.tb02875.x.

7. Gunn, J.K.L.; Rosales, C.B.; Center, K.E.; Nuñez, A.; Gibson, S.J.; Christ, C.; Ehiri, J.E. Prenatal Exposure to Cannabis and Maternal and Child Health Outcomes: A Systematic Review and Meta-Analysis. BMJ Open 2016, 6, e009986.

8. Conner, S.N.; Bedell, V.; Lipsey, K.; Macones, G.A.; Cahill, A.G.; Tuuli, M.G. Maternal Marijuana Use and Adverse Neonatal Outcomes: A Systematic Review and Meta-Analysis. Obstetrics and Gynecology 2016, 128, 713-723.

9. Michalski, C.A.; Hung, R.J.; Seeto, R.A.; Dennis, C.L.; Brooks, J.D.; Henderson, J.; Levitan, R.; Lye, S.J.; Matthews, S.G.; Knight, J.A. Association between Maternal Cannabis Use and Birth Outcomes: An Observational Study. BMC Pregnancy and Childbirth 2020, 20, doi:10.1186/s12884-020-03371-3.

10. Silvestri, C.; Di Marzo, V. The Endocannabinoid System in Energy Homeostasis and the Etiopathology of Metabolic Disorders. Cell Metabolism 2013, 17, 475-490.

11. Bouchard, J.F.; Lépicier, P.; Lamontagne, D. Contribution of Endocannabinoids in the Endothelial Protection Afforded by Ischemic Preconditioning in the Isolated Rat Heart. Life Sciences 2003, 72, 1859-1870, doi:10.1016/S0024-3205(02)02474-8.

12. Malenczyk, K.; Keimpema, E.; Piscitelli, F.; Calvigioni, D.; Björklund, P.; Mackie, K.; Marzo, V. Di; Hökfelt, T.G.M.; Dobrzyn, A.; Harkany, T. Fetal Endocannabinoids Orchestrate the Organization of Pancreatic Islet Microarchitecture. Proceedings of the National Academy of Sciences of the United States of America 2015, 112, E6185-E6194, doi:10.1073/pnas.1519040112.

13. Sun, X.; Dey, S.K. Endocannabinoid Signaling in Female Reproduction. ACS Chem. Neurosci 2012, 3, 349-355.

14. Ramírez-López, M.T.; Arco, R.; Decara, J.; Vázquez, M.; Blanco, R.N.; Alén, F.; Suárez, J.; De Heras, R.G.; De Fonseca, F.R. Exposure to a Highly Caloric Palatable Diet during the Perinatal Period Affects the Expression of the Endogenous Cannabinoid System in the Brain, Liver and Adipose Tissue of Adult Rat Offspring. PLoS ONE 2016, 11, doi:10.1371/journal.pone.0165432.

15. Maia, J.; Midão, L.; Cunha, S.C.; Almada, M.; Fonseca, B.M.; Braga, J.; Gonçalves, D.; Teixeira, N.; Correia-da-Silva, G. Effects of Cannabis Tetrahydrocannabinol on Endocannabinoid Homeostasis in Human Placenta. Arch Toxicol 2019, 93, 649-658, doi:10.1007/s00204-019-02389-7.

16. Fried, P.A. Short and Long-Term Effects of Pre-Natal Cannabis Inhalation upon Rat Offspring. Psychopharmacology 1976, 50, 285-291, doi:10.1007/BF00426846. 
17. Harbison, R.D.; Mantilla-Plata, B. Prenatal toxicity, maternal distribution and placental transfer of tetrahydrocannabinol. Journal of Pharmacology and Experimental Therapeutics 1972, 180.

18. Hurd, Y.L.; Wang, X.; Anderson, V.; Beck, O.; Minkoff, H.; Dow-Edwards, D. Marijuana Impairs Growth in Mid-Gestation Fetuses. Neurotoxicology and Teratology 2005, 27, 221-229, doi:10.1016/j.ntt.2004.11.002.

19. Benevenuto, S.G.; Domenico, M.D.; Martins, M.A.G.; Costa, N.S.; de Souza, A.R.L.; Costa, J.L.; Tavares, M.F.M.; Dolhnikoff, M.; Veras, M.M. Recreational Use of Marijuana during Pregnancy and Negative Gestational and Fetal Outcomes: An Experimental Study in Mice. Toxicology 2017, 376, 94-101, doi:10.1016/j.tox.2016.05.020.

20. Chang, X.; Bian, Y.; He, Q.; Yao, J.; Zhu, J.; Wu, J.; Wang, K.; Duan, T. Suppression of STAT3 Signaling by $\Delta 9$ Tetrahydrocannabinol (THC) Induces Trophoblast Dysfunction. Cellular Physiology and Biochemistry 2017, 42, 537-550, doi:10.1159/000477603.

21. Natale, B. V.; Gustin, K.N.; Lee, K.; Holloway, A.C.; Laviolette, S.R.; Natale, D.R.C.; Hardy, D.B. $\Delta$ 9-Tetrahydrocannabinol Exposure During Rat Pregnancy Leads To Symmetrical Fetal Growth Restriction and Labyrinth-Specific Vascular Defects in the Placenta. Scientific Reports 2020, 10, 1-15, doi:10.1038/s41598-019-57318-6.

22. James, P.T.; Rigby, N.; Leach, R. The Obesity Epidemic, Metabolic Syndrome and Future Prevention Strategies. European Journal of Cardiovascular Prevention E Rehabilitation 2004, 11, 3-8, doi:10.1097/01.hjr.0000114707.27531.48.

23. Eriksson, J.; Forsén, T.; Tuomilehto, J.; Osmond, C.; Barker, D. Size at Birth, Childhood Growth and Obesity in Adult Life. International Journal of Obesity 2001, 25, 735-740, doi:10.1038/sj.ijo.0801602.

24. Suomela, E.; Oikonen, M.; Pitkänen, N.; Ahola-Olli, A.; Virtanen, J.; Parkkola, R.; Jokinen, E.; Laitinen, T.; Hutri-Kähönen, N.; Kähönen, M.; et al. Childhood Predictors of Adult Fatty Liver. The Cardiovascular Risk in Young Finns Study. Journal of Hepatology 2016, 65, 784-790, doi:10.1016/j.jhep.2016.05.020.

25. Nobili, V.; Marcellini, M.; Marchesini, G.; Vanni, E.; Manco, M.; Villani, A.; Bugianesi, E. Intrauterine Growth Retardation, Insulin Resistance, and Nonalcoholic Fatty Liver Disease in Children. 2007, doi:10.2337/dc07-0281.

26. Ravelli, G.P.; Stein, Z.A.; Susser, M.W. Obesity in Young Men after Famine Exposure in Utero and Early Infancy. The New England journal of medicine 1976, 295, 349-353, doi:10.1056/NEJM197608122950701.

27. Yang, Z.; Zhao, W.; Zhang, X.; Mu, R.; Zhai, Y.; Kong, L.; Chen, C. Impact of Famine during Pregnancy and Infancy on Health in Adulthood. Obes Rev. 2008, 9, 95-9.

28. Faienza, M.F.; Brunetti, G.; Ventura, A.; D'Aniello, M.; Pepe, T.; Giordano, P.; Monteduro, M.; Cavallo, L. Nonalcoholic Fatty Liver Disease in Prepubertal Children Born Small for Gestational Age: Influence of Rapid Weight Catch-Up Growth. HRP 2013, 79, 103-109, doi:10.1159/000347217.

29. Barker, D.J.; Martyn, C.N.; Osmond, C.; Hales, C.N.; Fall, C.H. Growth in Utero and Serum Cholesterol Concentrations in Adult Life. BMJ (Clinical research ed.) 1993, 307, 1524-1527.

30. Ma, N.; Nicholson, C.J.; Wong, M.; Holloway, A.C.; Hardy, D.B. Fetal and Neonatal Exposure to Nicotine Leads to Augmented Hepatic and Circulating Triglycerides in Adult Male Offspring Due to Increased Expression of Fatty Acid Synthase. Toxicology and applied pharmacology 2013, 275, 1-11, doi:10.1016/j.taap.2013.12.010;

31. Sohi, G.; Marchand, K.; Revesz, A.; Arany, E.; Hardy, D.B. Maternal Protein Restriction Elevates Cholesterol in Adult Rat Offspring Due to Repressive Changes in Histone Modifications at the Cholesterol 7alpha-Hydroxylase Promoter. Molecular endocrinology (Baltimore, Md.) 2011, 25, 785-798, doi:10.1210/me.2010-0395.

32. Jensen-Urstad, A.P.; Semenkovich, C.F. Fatty Acid Synthase and Liver Triglyceride Metabolism: Housekeeper or Messenger? Biochimica et biophysica acta 2012, 1821, 747-753, doi:10.1016/j.bbalip.2011.09.017;

33. Kim, K.H. Regulation of Mammalian Acetyl-Coenzyme A Carboxylase. Annual Review of Nutrition 1997, 17, 77-99, doi:10.1146/annurev.nutr.17.1.77.

34. Miyazaki, M.; Ntambi, J.M. Role of Stearoyl-Coenzyme A Desaturase in Lipid Metabolism. Prostaglandins, leukotrienes, and essential fatty acids 2003, 68, 113-121. 
35. Cases, S.; Smith, S.J.; Zheng, Y.W.; Myers, H.M.; Lear, S.R.; Sande, E.; Novak, S.; Collins, C.; Welch, C.B.; Lusis, A.J.; et al. Identification of a Gene Encoding an Acyl CoA:Diacylglycerol Acyltransferase, a Key Enzyme in Triacylglycerol Synthesis. Proceedings of the National Academy of Sciences of the United States of America 1998, 95, 13018-13023.

36. Smith, S.J.; Cases, S.; Jensen, D.R.; Chen, H.C.; Sande, E.; Tow, B.; Sanan, D.A.; Raber, J.; Eckel, R.H.; Farese Jr, R. V Obesity Resistance and Multiple Mechanisms of Triglyceride Synthesis in Mice Lacking Dgat. Nature genetics 2000, 25, 87-90, doi:10.1038/75651.

37. Deodati, A.; Argemí, J.; Germani, D.; Puglianiello, A.; Alisi, A.; De Stefanis, C.; Ferrero, R.; Nobili, V.; Aragón, T.; Cianfarani, S. The Exposure to Uteroplacental Insufficiency Is Associated with Activation of Unfolded Protein Response in Postnatal Life. PLOS ONE 2018, 13, e0198490, doi:10.1371/journal.pone.0198490.

38. Yamada, M.; Wolfe, D.; Han, G.; French, S.W.; Ross, M.G.; Desai, M. Early Onset of Fatty Liver in Growth-Restricted Rat Fetuses and Newborns. Congenital Anomalies 2011, 51, 167-173, doi:10.1111/j.1741-4520.2011.00336.x.

39. Cheng, K.; Ji, S.; Jia, P.; Zhang, H.; Wang, T.; Song, Z.; Zhang, L.; Wang, T. Resveratrol Improves Hepatic Redox Status and Lipid Balance of Neonates with Intrauterine Growth Retardation in a Piglet Model. BioMed Research International 2020, 2020, doi:10.1155/2020/7402645.

40. Wang, J.; Chen, L.; Li, D.; Yin, Y.; Wang, X.; Li, P.; Dangott, L.J.; Hu, W.; Wu, G. Intrauterine Growth Restriction Affects the Proteomes of the Small Intestine, Liver, and Skeletal Muscle in Newborn Pigs. Journal of Nutrition 2008, 138, 60-66, doi:10.1093/jn/138.1.60.

41. Devarajan, A.; Rajasekaran, N.S.; Valburg, C.; Ganapathy, E.; Bindra, S.; Freije, W.A. Maternal Perinatal Calorie Restriction Temporally Regulates the Hepatic Autophagy and Redox Status in Male Rat. Free Radical Biology and Medicine 2019, doi:10.1016/j.freeradbiomed.2018.09.029.

42. Moraes, C.; Rebelato, H.J.; Amaral, M.E.C.; Resende, T.M.; Silva, E.V.C.; Esquisatto, M.A.M.; Catisti, R. Effect of Maternal Protein Restriction on Liver Metabolism in Rat Offspring. The Journal of Physiological Sciences 2014, 64, 347-355, doi:10.1007/s12576-014-0325-8.

43. Theys, N.; Clippe, A.; Bouckenooghe, T.; Reusens, B.; Remacle, C. Early Low Protein Diet Aggravates Unbalance between Antioxidant Enzymes Leading to Islet Dysfunction. PLoS ONE 2009, 4, e6110, doi:10.1371/journal.pone.0006110.

44. Park, K.S.; Kim, S.K.; Kim, M.S.; Cho, E.Y.; Lee, J.H.; Lee, K.-U.; Pak, Y.K.; Lee, H.K. Fetal and Early Postnatal Protein Malnutrition Cause Long-Term Changes in Rat Liver and Muscle Mitochondria. The Journal of Nutrition 2003, 133, 3085-3090, doi:10.1093/jn/133.10.3085.

45. Oke, S.L.; Sohi, G.; Hardy, D.B. Perinatal Protein Restriction with Postnatal Catch-up Growth Leads to Elevated P66Shc and Mitochondrial Dysfunction in the Adult Rat Liver. Reproduction 2020, 159, 27-39.

46. Giorgio, M.; Migliaccio, E.; Orsini, F.; Paolucci, D.; Moroni, M.; Contursi, C.; Pelliccia, G.; Luzi, L.; Minucci, S.; Marcaccio, M.; et al. Electron Transfer between Cytochrome c and P66Shcgenerates Reactive Oxygen Species That Trigger Mitochondrial Apoptosis. Cell 2005, doi:10.1016/j.cell.2005.05.011.

47. Berniakovich, I.; Trinei, M.; Stendardo, M.; Migliaccio, E.; Minucci, S.; Bernardi, P.; Pelicci, P.G.; Giorgio, M. P66ShcGenerated Oxidative Signal Promotes Fat Accumulation. Journal of Biological Chemistry 2008, 283, 34283-34293, doi:10.1074/jbc.M804362200.

48. Tomita, K.; Teratani, T.; Suzuki, T.; Oshikawa, T.; Yokoyama, H.; Shimamura, K.; Nishiyama, K.; Mataki, N.; Irie, R.; Minamino, T.; et al. P53/P66Shc-Mediated Signaling Contributes to the Progression of Non-Alcoholic Steatohepatitis in Humans and Mice. Journal of Hepatology 2012, 57, 837-843, doi:10.1016/j.jhep.2012.05.013.

49. Luyckx, V.A.; Compston, C.A.; Simmen, T.; Mueller, T.F. Accelerated Senescence in Kidneys of Low-Birth-Weight Rats after Catch-up Growth. American Journal of Physiology-Renal Physiology 2009, 297, F1697-F1705, doi:10.1152/ajprenal.00462.2009.

50. Raez-Villanueva, S.; Debnath, A.; Hardy, D.B.; Holloway, A.C. Prenatal Nicotine Exposure Leads to Decreased Histone H3 Lysine 9 (H3K9) Methylation and Increased P66shc Expression in the Neonatal Pancreas. Journal of Developmental Origins of Health and Disease 2021, 28, 1-5. 
51. Sohi, G.; Revesz, A.; Ramkumar, J.; Hardy, D.B. Higher Hepatic MiR-29 Expression in Undernourished Male Rats During the Postnatal Period Targets the Long-Term Repression of IGF-1. Endocrinology 2015, 156, 3069-3076, doi:10.1210/EN.2015-1058.

52. Barra, N.G.; Lisyansky, M.; Vanduzer, T.A.; Raha, S.; Holloway, A.C.; Hardy, D.B. Maternal Nicotine Exposure Leads to Decreased Cardiac Protein Disulfide Isomerase and Impaired Mitochondrial Function in Male Rat Offspring. Journal of Applied Toxicology 2017, 37, 1517-1526, doi:10.1002/jat.3503.

53. Ipsen, D.H.; Lykkesfeldt, J.; Tveden-Nyborg, P. Molecular Mechanisms of Hepatic Lipid Accumulation in Non-Alcoholic Fatty Liver Disease. Cell Mol Life Sci 2018, 75, 3313-3327, doi:10.1007/s00018-018-2860-6.

54. Lone, A.; Harris, R.A.; Singh, O.; Betts, D.H.; Cumming, R.C. P66Shc Activation Promotes Increased Oxidative Phosphorylation and Renders CNS Cells More Vulnerable to Amyloid Beta Toxicity. Scientific Reports 2018, 8, 17081-17081, doi:10.1038/s41598-018-35114-y.

55. Brand, M.D. The Sites and Topology of Mitochondrial Superoxide Production. Exp Gerontol 2010, 45, 466-472, doi:10.1016/j.exger.2010.01.003.

56. Wang, Z.; Zhao, Y.; Zhao, H.; Zhou, J.; Feng, D.; Tang, F.; Li, Y.; Lv, L.; Chen, Z.; Ma, X.; et al. Inhibition of P66Shc Oxidative Signaling via CA-Induced Upregulation of MiR-203a-3p Alleviates Liver Fibrosis Progression. Molecular Therapy - Nucleic Acids 2020, 21, 751-763, doi:10.1016/j.omtn.2020.07.013.

57. Musa, M.G.; Kagura, J.; Pisa, P.T.; Norris, S.A. Relationship between Early Growth and CVD Risk Factors in Adolescents. Journal of Developmental Origins of Health and Disease 2015, 7, 132-143, doi:10.1017/S2040174415007953.

58. Victora, C.G.; Barros, F.C.; Lima, R.C.; Behague, D.P.; Gon alves, H.; Horta, B.L.; Gigante, D.P.; Vaughan, J.P. The Pelotas Birth Cohort Study, Rio Grande Do Sul, Brazil, 1982-2001. Cadernos de saúde pública / Ministério da Saúde, Fundação Oswaldo Cruz, Escola Nacional de Saúde Pública 2003, 19, 1241-1256, doi:10.1590/S0102-311X2003000500003.

59. Perng, W.; Hajj, H.; Belfort, M.B.; Rifas-Shiman, S.L.; Kramer, M.S.; Gillman, M.W.; Oken, E. Birth Size, Early Life Weight Gain, and Midchildhood Cardiometabolic Health. Journal of Pediatrics 2016, 173, 122-130.e1, doi:10.1016/j.jpeds.2016.02.053.

60. Gao, Y.J.; Holloway, A.C.; Zeng, Z.H.; Lim, G.E.; Petrik, J.J.; Foster, W.G.; Lee, R.M. Prenatal Exposure to Nicotine Causes Postnatal Obesity and Altered Perivascular Adipose Tissue Function. Obesity research 2005, 13, 687-692, doi:10.1038/oby.2005.77.

61. Guan, H.; Arany, E.; van Beek, J.P.; Chamson-Reig, A.; Thyssen, S.; Hill, D.J.; Yang, K. Adipose Tissue Gene Expression Profiling Reveals Distinct Molecular Pathways That Define Visceral Adiposity in Offspring of Maternal Protein-Restricted Rats. American journal of physiology.Endocrinology and metabolism 2005, 288, E663-73, doi:10.1152/ajpendo.00461.2004.

62. Riediger, N.D.; Clara, I. Prevalence of Metabolic Syndrome in the Canadian Adult Population. CMAJ: Canadian Medical Association journal = journal de l'Association medicale canadienne 2011, 183, E1127-34, doi:10.1503/cmaj.110070;

63. Gillies, R.; Lee, K.; Vanin, S.; Laviolette, S.R.; Holloway, A.C.; Arany, E.; Hardy, D.B. Maternal Exposure to $\Delta 9$ Tetrahydrocannabinol Impairs Female Offspring Glucose Homeostasis and Endocrine Pancreatic Development in the Rat. Reprod Toxicol 2020, 94, 84-91, doi:10.1016/j.reprotox.2020.04.070.

64. Villa, A.; Della Torre, S.; Stell, A.; Cook, J.; Brown, M.; Maggi, A. Tetradian Oscillation of Estrogen Receptor $\alpha$ Is Necessary to Prevent Liver Lipid Deposition. Proc Natl Acad Sci U S A 2012, 109, 11806-11811, doi:10.1073/pnas.1205797109.

65. Park, C.J.; Zhao, Z.; Glidewell-Kenney, C.; Lazic, M.; Chambon, P.; Krust, A.; Weiss, J.; Clegg, D.J.; Dunaif, A.; Jameson, J.L.; et al. Genetic Rescue of Nonclassical ER $\alpha$ Signaling Normalizes Energy Balance in Obese Er $\alpha$-Null Mutant Mice. J Clin Invest 2011, 121, 604-612, doi:10.1172/JCI41702.

66. Browning, J.D.; Szczepaniak, L.S.; Dobbins, R.; Nuremberg, P.; Horton, J.D.; Cohen, J.C.; Grundy, S.M.; Hobbs, H.H. Prevalence of Hepatic Steatosis in an Urban Population in the United States: Impact of Ethnicity. Hepatology 2004, 40, 13871395, doi:10.1002/hep.20466.

67. Hamaguchi, M.; Kojima, T.; Takeda, N.; Nakagawa, T.; Taniguchi, H.; Fujii, K.; Omatsu, T.; Nakajima, T.; Sarui, H.; Shimazaki, M.; et al. The Metabolic Syndrome as a Predictor of Nonalcoholic Fatty Liver Disease. Ann Intern Med 2005, 143, 722-728, doi:10.7326/0003-4819-143-10-200511150-00009. 
68. Suzuki, A.; Angulo, P.; Lymp, J.; St Sauver, J.; Muto, A.; Okada, T.; Lindor, K. Chronological Development of Elevated Aminotransferases in a Nonalcoholic Population. Hepatology 2005, 41, 64-71, doi:10.1002/hep.20543.

69. Tsuneto, A.; Hida, A.; Sera, N.; Imaizumi, M.; Ichimaru, S.; Nakashima, E.; Seto, S.; Maemura, K.; Akahoshi, M. Fatty Liver Incidence and Predictive Variables. Hypertens Res 2010, 33, 638-643, doi:10.1038/hr.2010.45.

70. Sung, K.-C.; Kim, B.-S.; Cho, Y.-K.; Park, D.; Woo, S.; Kim, S.; Wild, S.H.; Byrne, C.D. Predicting Incident Fatty Liver Using Simple Cardio-Metabolic Risk Factors at Baseline. BMC Gastroenterology 2012, 12, 84, doi:10.1186/1471-230X-12-84.

71. Pramfalk, C.; Pavlides, M.; Banerjee, R.; McNeil, C.A.; Neubauer, S.; Karpe, F.; Hodson, L. Sex-Specific Differences in Hepatic Fat Oxidation and Synthesis May Explain the Higher Propensity for NAFLD in Men. J Clin Endocrinol Metab 2015, 100, 44254433, doi:10.1210/jc.2015-2649.

72. Tran, C.; Jacot-Descombes, D.; Lecoultre, V.; Fielding, B.A.; Carrel, G.; Lê, K.-A.; Schneiter, P.; Bortolotti, M.; Frayn, K.N.; Tappy, L. Sex Differences in Lipid and Glucose Kinetics after Ingestion of an Acute Oral Fructose Load. Br J Nutr 2010, 104, 1139-1147, doi:10.1017/S000711451000190X.

73. Leamy, A.K.; Hasenour, C.M.; Egnatchik, R.A.; Trenary, I.A.; Yao, C.-H.; Patti, G.J.; Shiota, M.; Young, J.D. Knockdown of Triglyceride Synthesis Does Not Enhance Palmitate Lipotoxicity or Prevent Oleate-Mediated Rescue in Rat Hepatocytes. Biochim Biophys Acta 2016, 1861, 1005-1014, doi:10.1016/j.bbalip.2016.05.013.

74. Elmes, M.W.; Kaczocha, M.; Berger, W.T.; Leung, K.; Ralph, B.P.; Wang, L.; Sweeney, J.M.; Miyauchi, J.T.; Tsirka, S.E.; Ojima, I.; et al. Fatty Acid-Binding Proteins (FABPs) Are Intracellular Carriers for $\Delta 9$-Tetrahydrocannabinol (THC) and Cannabidiol (CBD)*. Journal of Biological Chemistry 2015, 290, 8711-8721, doi:10.1074/jbc.M114.618447.

75. Elmes, M.W.; Prentis, L.E.; McGoldrick, L.L.; Giuliano, C.J.; Sweeney, J.M.; Joseph, O.M.; Che, J.; Carbonetti, G.S.; Studholme, K.; Deutsch, D.G.; et al. FABP1 Controls Hepatic Transport and Biotransformation of $\Delta 9$-THC. Sci Rep 2019, 9, 7588, doi:10.1038/s41598-019-44108-3.

76. Mukai, T.; Egawa, M.; Takeuchi, T.; Yamashita, H.; Kusudo, T. Silencing of FABP1 Ameliorates Hepatic Steatosis, Inflammation, and Oxidative Stress in Mice with Nonalcoholic Fatty Liver Disease. FEBS Open Bio 2017, 7, 1009-1016, doi:10.1002/2211-5463.12240.

Rolo, A.P.; Teodoro, J.S.; Palmeira, C.M. Role of Oxidative Stress in the Pathogenesis of Nonalcoholic Steatohepatitis. Free Radical Biology and Medicine 2012, 52, 59-69.

78. Chen, Z.; Tian, R.; She, Z.; Cai, J.; Li, H. Role of Oxidative Stress in the Pathogenesis of Nonalcoholic Fatty Liver Disease. Free Radical Biology and Medicine 2020, 152, 116-141.

79. Mantena, S.K.; King, A.L.; Andringa, K.K.; Eccleston, H.B.; Bailey, S.M. Mitochondrial Dysfunction and Oxidative Stress in the Pathogenesis of Alcohol- and Obesity-Induced Fatty Liver Diseases. Free Radical Biology and Medicine 2008, 44, $1259-1272$.

80. Begriche, K.; Massart, J.; Robin, M.A.; Bonnet, F.; Fromenty, B. Mitochondrial Adaptations and Dysfunctions in Nonalcoholic Fatty Liver Disease. Hepatology 2013, 58, 1497-1507.

81. Seo, E.; Kang, H.; Choi, H.; Choi, W.; Jun, H.S. Reactive Oxygen Species-Induced Changes in Glucose and Lipid Metabolism Contribute to the Accumulation of Cholesterol in the Liver during Aging. Aging Cell 2019, 18, doi:10.1111/acel.12895.

Lockman, K.A.; Baren, J.P.; Pemberton, C.J.; Baghdadi, H.; Burgess, K.E.; Plevris-Papaioannou, N.; Lee, P.; Howie, F.; Beckett, G.; Pryde, A.; et al. Oxidative Stress Rather than Triglyceride Accumulation Is a Determinant of Mitochondrial Dysfunction in in Vitro Models of Hepatic Cellular Steatosis. Liver International 2012, 32, 1079-1092, doi:10.1111/j.1478-3231.2012.02775.x.

83. Migliaccio, E.; Giogio, M.; Mele, S.; Pelicci, G.; Reboldi, P.; Pandolfi, P.P.; Lanfrancone, L.; Pelicci, P.G. The P66(Shc) Adaptor Protein Controls Oxidative Stress Response and Life Span in Mammals. Nature 1999, doi:10.1038/46311.

84. Orsini, F.; Migliaccio, E.; Moroni, M.; Contursi, C.; Raker, V.A.; Piccini, D.; Martin-Padura, I.; Pelliccia, G.; Trinei, M.; Bono, M.; et al. The Life Span Determinant P66Shc Localizes to Mitochondria Where It Associates with Mitochondrial Heat Shock Protein 70 and Regulates Trans-Membrane Potential. Journal of Biological Chemistry 2004, doi:10.1074/jbc.M401844200.

85. Trinei, M.; Migliaccio, E.; Bernardi, P.; Paolucci, F.; Pelicci, P.; Giorgio, M. p66Shc, Mitochondria, and the Generation of Reactive Oxygen Species. Methods in enzymology 2013, 528, 99-110. 
86. Firmin, S.; Elmhiri, G.; Crepin, D.; Souidi, M.; Taouis, M.; Abdennebi-Najar, L. Formula Derived Maillard Reaction Products in Post-Weaning Intrauterine Growth-Restricted Piglets Induce Developmental Programming of Hepatic Oxidative Stress Independently of MicroRNA-21 and MicroRNA-155. J Dev Orig Health Dis 2018, 9, 566-572, doi:10.1017/S2040174417001015.

87. Zinkhan, E.K.; Yu, B.; McKnight, R. Uteroplacental Insufficiency Impairs Cholesterol Elimination in Adult Female GrowthRestricted Rat Offspring Fed a High-Fat Diet. Reprod Sci 2019, 26, 1173-1180, doi:10.1177/1933719118811649.

88. Saget, S.; Cong, R.; Decourtye, L.; Endale, M.-L.; Martinerie, L.; Girardet, C.; Perret, C.; Clemessy, M.; Leneuve, P.; Dinard, L.; et al. Changes in Circulating MiRNA19a-3p Precede Insulin Resistance Programmed by Intra-Uterine Growth Retardation in Mice. Mol Metab 2020, 42, 101083, doi:10.1016/j.molmet.2020.101083.

89. Roderburg, C.; Urban, G.-W.; Bettermann, K.; Vucur, M.; Zimmermann, H.; Schmidt, S.; Janssen, J.; Koppe, C.; Knolle, P.; Castoldi, M.; et al. Micro-RNA Profiling Reveals a Role for MiR-29 in Human and Murine Liver Fibrosis. Hepatology 2011, 53, 209-218, doi:10.1002/hep.23922.

90. Lin, H.-Y.; Wang, F.-S.; Yang, Y.-L.; Huang, Y.-H. MicroRNA-29a Suppresses CD36 to Ameliorate High Fat Diet-Induced Steatohepatitis and Liver Fibrosis in Mice. Cells 2019, 8, doi:10.3390/cells8101298.

91. Yang, Y.-L.; Kuo, H.-C.; Wang, F.-S.; Huang, Y.-H. MicroRNA-29a Disrupts DNMT3b to Ameliorate Diet-Induced NonAlcoholic Steatohepatitis in Mice. Int J Mol Sci 2019, 20, doi:10.3390/ijms20061499.

92. Lee, K.; Laviolette, S.R.; Hardy, D.B. Exposure to $\Delta$ 9-Tetrahydrocannabinol during Rat Pregnancy Leads to Impaired Cardiac Dysfunction in Postnatal Life. Pediatr Res 2021, 1-8, doi:10.1038/s41390-021-01511-9.

93. Grundy, D. Principles and Standards for Reporting Animal Experiments in The Journal of Physiology and Experimental Physiology. Exp Physiol 2015, 100, 755-758, doi:10.1113/EP085299.

94. Kilkenny, C.; Browne, W.J.; Cuthill, I.C.; Emerson, M.; Altman, D.G. Improving Bioscience Research Reporting: The ARRIVE Guidelines for Reporting Animal Research. PLOS Biology 2010, 8, e1000412, doi:10.1371/journal.pbio.1000412.

95. Klein, C.; Karanges, E.; Spiro, A.; Wong, A.; Spencer, J.; Huynh, T.; Gunasekaran, N.; Karl, T.; Long, L.E.; Huang, X.-F.; et al. Cannabidiol Potentiates $\Delta^{9}$-Tetrahydrocannabinol (THC) Behavioural Effects and Alters THC Pharmacokinetics during Acute and Chronic Treatment in Adolescent Rats. Psychopharmacology (Berl) 2011, 218, 443-457, doi:10.1007/s00213-011-23420.

96. Falcon, M.; Pichini, S.; Joya, J.; Pujadas, M.; Sanchez, A.; Vall, O.; García Algar, O.; Luna, A.; de la Torre, R.; Rotolo, M.C.; et al. Maternal Hair Testing for the Assessment of Fetal Exposure to Drug of Abuse during Early Pregnancy: Comparison with Testing in Placental and Fetal Remains. Forensic Sci Int 2012, 218, 92-96, doi:10.1016/j.forsciint.2011.10.022.

97. Schwope, D.M.; Karschner, E.L.; Gorelick, D.A.; Huestis, M.A. Identification of Recent Cannabis Use: Whole-Blood and Plasma Free and Glucuronidated Cannabinoid Pharmacokinetics Following Controlled Smoked Cannabis Administration. Clinical chemistry 2011, 57, 1406-14, doi:10.1373/clinchem.2011.171777.

98. Mato, S.; Chevaleyre, V.; Robbe, D.; Pazos, A.; Castillo, P.E.; Manzoni, O.J. A Single In-Vivo Exposure to $\Delta 9 \mathrm{THC}$ Blocks Endocannabinoid-Mediated Synaptic Plasticity. Nature Neuroscience 2004, 7, 585-586, doi:10.1038/nn1251.

99. Tortoriello, G.; Morris, C.V.; Alpar, A.; Fuzik, J.; Shirran, S.L.; Calvigioni, D.; Keimpema, E.; Botting, C.H.; Reinecke, K.; Herdegen, T.; et al. Miswiring the Brain: $\Delta 9$-Tetrahydrocannabinol Disrupts Cortical Development by Inducing an SCG10/Stathmin-2 Degradation Pathway. EMBO J 2014, 33, 668-685, doi:10.1002/embj.201386035.

100. Dinieri, J.A.; Hurd, Y.L. Rat Models of Prenatal and Adolescent Cannabis Exposure. Methods Mol Biol 2012, 829, 231-242, doi:10.1007/978-1-61779-458-2_14.

101. Hayes, E.K.; Lechowicz, A.; Petrik, J.J.; Storozhuk, Y.; Paez-Parent, S.; Dai, Q.; Samjoo, I.A.; Mansell, M.; Gruslin, A.; Holloway, A.C.; et al. Adverse Fetal and Neonatal Outcomes Associated with a Life-Long High Fat Diet: Role of Altered Development of the Placental Vasculature. PloS one 2012, 7, e33370-e33370, doi:10.1371/journal.pone.0033370;

102. Beamish, C.A.; Zhang, L.; Szlapinski, S.K.; Strutt, B.J.; Hill, D.J. An Increase in Immature $\beta$-Cells Lacking Glut2 Precedes the Expansion of $\beta$-Cell Mass in the Pregnant Mouse. PLoS One 2017, 12, e0182256, doi:10.1371/journal.pone.0182256. 